\title{
MOLECULAR GAS IN LENSED $z>2$ QUASAR HOST GALAXIES AND THE STAR FORMATION LAW FOR GALAXIES WITH LUMINOUS ACTIVE GALACTIC NUCLEI
}

\author{
DOMINIK A. RIECHERs ${ }^{1}$ \\ Astronomy Department, California Institute of Technology, MC 249-17, 1200 East California Boulevard, Pasadena, CA 91125, USA; dr@ caltech.edu \\ Received 2010 November 24; accepted 2011 January 28; published 2011 March 10
}

\begin{abstract}
We report the detection of luminous $\mathrm{CO}(J=2 \rightarrow 1), \mathrm{CO}(J=3 \rightarrow 2)$, and $\mathrm{CO}(J=4 \rightarrow 3)$ emission in the strongly lensed high-redshift quasars B1938+666 $(z=2.059)$, HE 0230-2130 $(z=2.166)$, HE 1104-1805 $(z=2.322)$, and B1359+154 $(z=3.240)$, using the Combined Array for Research in Millimeter-wave Astronomy. B1938+666 was identified in a "blind" CO redshift search, demonstrating the feasibility of such investigations with millimeter interferometers. These galaxies are lensing-amplified by factors of $\mu_{\mathrm{L}} \simeq 11-170$, and thus allow us to probe the molecular gas in intrinsically fainter galaxies than currently possible without the aid of gravitational lensing. We report lensing-corrected intrinsic $\mathrm{CO}$ line luminosities of $L_{\mathrm{CO}}^{\prime}=0.65-21 \times 10^{9} \mathrm{~K} \mathrm{~km} \mathrm{~s}^{-1} \mathrm{pc}^{2}$, translating to $\mathrm{H}_{2}$ masses of $M\left(\mathrm{H}_{2}\right)=0.52-17 \times 10^{9}\left(\alpha_{\mathrm{CO}} / 0.8\right) M_{\odot}$. To investigate whether or not the active galactic nucleus (AGN) in luminous quasars substantially contributes to $L_{\mathrm{FIR}}$, we study the $L_{\mathrm{CO}}^{\prime}-L_{\mathrm{FIR}}$ relation for quasars relative to galaxies without a luminous AGN as a function of redshift. We find no substantial differences between submillimeter galaxies and high- $z$ quasars, but marginal evidence for an excess in $L_{\mathrm{FIR}}$ in nearby low- $L_{\mathrm{FIR}}$ AGN galaxies. This may suggest that an AGN contribution to $L_{\mathrm{FIR}}$ is significant in systems with relatively low gas and dust content, but only minor in the most far-infrared-luminous galaxies (in which $L_{\mathrm{FIR}}$ is dominated by star formation).
\end{abstract}

Key words: cosmology: observations - galaxies: active - galaxies: formation - galaxies: high-redshift - galaxies: starburst - radio lines: galaxies

Online-only material: color figures

\section{INTRODUCTION}

Molecular line emission from galaxies at high redshift $(z>2)$ has proven to be a unique tool to investigate the stellar mass buildup in the often heavily dust-enshrouded star-forming environments of massive galaxies at early cosmic times. Molecular gas (CO) was detected in $26 z>2$ quasar host galaxies to date (see, e.g., review by Solomon \& Vanden Bout 2005), all of which are luminous in the rest-frame far-infrared (FIR; typically $L_{\mathrm{FIR}} \gtrsim 10^{13} L_{\odot}$; e.g., Wang et al. 2008). These studies typically reveal molecular gas masses of few times $10^{10} M_{\odot}$, indicating gas-rich host galaxies for the luminous active galactic nuclei (AGNs) that are observed at optical wavelengths. The high FIR luminosities are thought to be dominated by dust heating from young stars formed in intense starburst events comparable to those found in submillimeter galaxies (SMGs), with star formation rates (SFRs) exceeding $1000 M_{\odot} \mathrm{yr}^{-1}$. These starbursts are fueled by the massive molecular gas reservoirs, which can maintain them for $>10^{7} \mathrm{yr}$ (e.g., Riechers et al. 2008). The connection between gas mass and star formation is reflected in the relation between CO and FIR luminosities (e.g., Sanders et al. 1991; Gao \& Solomon 2004), which in turn can be used to constrain the contribution of the AGN to $L_{\mathrm{FIR}}$ in quasars (e.g., Riechers et al. 2006). The presence of both luminous AGNs and massive starbursts makes these objects the ideal candidates to better understand the AGN-starburst connection back to early cosmic times.

The currently most efficient way to investigate the molecular gas properties of quasar host galaxies with somewhat less extreme star formation events is by facilitating the flux magnification provided by gravitational lensing. Besides the flux boost, the spatial magnification of lensed quasars also aids in resolving

\footnotetext{
1 Hubble Fellow.
}

these systems, allowing us to probe the gas reservoirs at smaller physical scales. About one-third of the CO-detected $z>2$ quasar host galaxies are gravitationally lensed (nine sources), with typical magnification factors between a few and $\sim 30$ (Solomon \& Vanden Bout 2005). To study the gas properties of high-redshift AGN host galaxies in more detail, we here enhance this sample by four galaxies with magnification factors of 10.8-173, including double, quadruple, sextuple, and Einstein ring lens configurations.

In this paper, we report the detection of $\mathrm{CO}(J=2 \rightarrow 1)$, $\mathrm{CO}(J=3 \rightarrow 2)$, and $\mathrm{CO}(J=4 \rightarrow 3)$ emission in the far-infraredluminous, strongly lensed quasar host galaxies of B1938+666 $(z=2.059)$, HE $0230-2130(z=2.166)$, HE 1104-1805 $(z=$ $2.322)$, and B1359+154 ( $z=3.240)$, using the Combined Array for Research in Millimeter-wave Astronomy (CARMA). We use a concordance, flat $\Lambda \mathrm{CDM}$ cosmology throughout, with $H_{0}=$ $71 \mathrm{~km} \mathrm{~s}^{-1} \mathrm{Mpc}^{-1}, \Omega_{\mathrm{M}}=0.27$, and $\Omega_{\Lambda}=0.73$ (Spergel et al. 2003, 2007).

\section{OBSERVATIONS}

\subsection{Targeted CO Searches}

We used CARMA to observe the $\mathrm{CO}(J=3 \rightarrow 2)$ transition line $\left(v_{\text {rest }}=345.7960 \mathrm{GHz}\right)$ toward the $z>2$ quasars HE 0230-2130, HE 1104-1805, and B1359+154, and the $\mathrm{CO}(J=4 \rightarrow 3)$ line $\left(v_{\text {rest }}=461.0408 \mathrm{GHz}\right)$ toward B1359+154, all of which are redshifted to the $3 \mathrm{~mm}$ atmospheric window (see Table 1 for redshifted frequencies). All targets were observed with 14 or 15 antennas (corresponding to 91 or 105 baselines per antenna configuration) for a total of 14 tracks in the C, D, and E configurations between 2009 June 29 and 2010 March 22 , amounting to a total observing time of $57 \mathrm{hr}(33 \mathrm{hr}$ on source). 
Table 1

Summary of Observations

\begin{tabular}{|c|c|c|c|c|c|c|c|c|c|}
\hline Target & Line & $z_{\mathrm{opt}}$ & $\begin{array}{c}v_{\text {obs }}{ }^{\mathrm{a}} \\
(\mathrm{GHz})\end{array}$ & Sideband & Configuration & Dates & Tracks & Calibrator & $\begin{array}{c}t_{\mathrm{on}} / t_{\mathrm{tot}} \mathrm{b} \\
(\mathrm{hr})\end{array}$ \\
\hline B1938+666 & $\begin{array}{l}\mathrm{CO}(J=2 \rightarrow 1) \\
\mathrm{CO}(J=3 \rightarrow 2)\end{array}$ & $\cdots$ & $\begin{array}{r}75.3638 \\
113.0425\end{array}$ & & $\mathrm{C} / \mathrm{D} / \mathrm{E}$ & 2010 Jul 5-Oct 10 & $3 / 10 / 4$ & $1849+670$ & $43.6 / 69.9$ \\
\hline HE 0230-2130 & $\mathrm{CO}(J=3 \rightarrow 2)$ & 2.163 & 109.360 & USB & $\mathrm{D}$ & 2009 Aug 1-Sep 02 & 5 & $\begin{array}{l}0132-169 \\
0204-170\end{array}$ & $13.3 / 22.9$ \\
\hline HE 1104-1805 & $\mathrm{CO}(J=3 \rightarrow 2)$ & 2.319 & 104.155 & USB & $\mathrm{C}$ & 2010 Mar $21 / 22$ & 2 & $1127-189$ & $6.6 / 10.6$ \\
\hline B1359+154 & $\begin{array}{l}\mathrm{CO}(J=3 \rightarrow 2) \\
\mathrm{CO}(J=4 \rightarrow 3)\end{array}$ & 3.235 & $\begin{array}{r}81.652 \\
108.864 \\
\end{array}$ & $\begin{array}{l}\text { LSB } \\
\text { USB }\end{array}$ & $\begin{array}{l}\mathrm{D} / \mathrm{E} \\
\mathrm{D} / \mathrm{E}\end{array}$ & $\begin{array}{l}2009 \text { Jul 7-Aug } 15 \\
2009 \text { Jun 29-Jul } 27\end{array}$ & $\begin{array}{l}1 / 2 \\
2 / 2\end{array}$ & $1357+193$ & $\begin{array}{l}5.9 / 10.6 \\
7.2 / 13.3 \\
\end{array}$ \\
\hline Total & & & & & & & 31 & & $76.6 / 127.3$ \\
\hline
\end{tabular}

Notes.

a Tuning frequency (where applicable), corresponding to zero velocity in Figures 1 and 7.

b Time on source/total.

Weather conditions scaled between acceptable and excellent for observations at $3 \mathrm{~mm}$ wavelengths. The nearby quasars J0132 - 169, J0204-170, J1127-189, and J1357+193 were observed every 15 minutes for secondary amplitude and phase calibration. The strong calibrator sources J0423-013, J1058+015, $3 \mathrm{C} 446$, and $3 \mathrm{C} 273$ were observed at least once per track for bandpass and secondary flux calibration. Absolute fluxes were bootstrapped relative to Mars, Uranus, MWC349, or 3C84 (when no planet was available). Pointing was performed at least every $2-4 \mathrm{hr}$ on nearby sources, using both radio and optical modes. The resulting total calibration is estimated to be accurate within $\sim 15 \%$.

All observations were carried out with the previous generation correlator. The $\mathrm{CO}(J=3 \rightarrow 2)$ line in $\mathrm{B} 1359+154$ was centered in the lower sideband (LSB). All other lines were centered in the upper sideband (USB) at intermediate frequencies of $2.5 \mathrm{GHz}$. Three bands with 15 channels of $31.25 \mathrm{MHz}\left(\sim 86-115 \mathrm{~km} \mathrm{~s}^{-1}\right)$ width each were centered on the tuning frequencies. The bands were overlapped by two channels to improve calibration of the correlated data set, leading to an effective bandwidth of 1281.25 MHz ( 3500-4700 $\mathrm{km} \mathrm{s}^{-1}$ ) per sideband.

For data reduction and analysis, the MIRIAD package was used. The final plots were created with the GILDAS package. All data were imaged using "natural' weighting, yielding synthesized beam sizes of $7^{\prime \prime} .3 \times 4$ ". 2,2 . $.5 \times 1^{\prime \prime} .9,10^{\prime \prime} .1 \times 7^{\prime \prime} .8$, and $9^{\prime \prime} .4 \times 6$. 8 for the $\mathrm{CO}(J=3 \rightarrow 2)$ observations in HE 0230-2130, $\mathrm{HE} 1104-1805$, and $\mathrm{B} 1359+154$ and the $\mathrm{CO}(J=4 \rightarrow 3)$ observations of $\mathrm{B} 1359+154$, respectively. The final $\mathrm{rms}$ noise values are $0.52,0.65,0.97$, and $0.71 \mathrm{mJy} \mathrm{beam}^{-1}$ over 1114,450 , 344 , and $430 \mathrm{~km} \mathrm{~s}^{-1}(406.25,156.25,93.75$, and $156.25 \mathrm{MHz})$. Averaging over all line-free data (LSB+USB) in the observations of B1359+154 (both line setups) yields a beam size of 9.' $8 \times 7^{\prime \prime} .3$ and an rms noise of $0.12 \mathrm{mJy}^{\text {beam }}{ }^{-1}$.

\section{2. "Blind" CO Search}

We also used CARMA to observe the very submillimeterbright, radio-loud quasar B1938+666, for which the redshift was previously unknown. Thus, we searched the entire frequency range from 82.4 to $115.3 \mathrm{GHz}$ for $\mathrm{CO}$ line emission, facilitating the large bandwidth of $3708.096 \mathrm{MHz}$ per sideband (eight bands with 95 channels of $5.208 \mathrm{MHz}$ with per sideband, overlapping bands by typically six channels to reduce sensitivity losses due to bandpass rolloff) of the new correlator. Observations were carried out for 14 tracks in the D and E configurations between 2010 July 05 and September 08, amounting to a total observing time of $58 \mathrm{hr}(37 \mathrm{hr}$ on source). A total of six (partially overlapping) frequency settings were used to cover the 82.4-115.3 GHz frequency range. Observations were typically carried out in pairs of frequency settings with an IF frequency of $3.6 \mathrm{GHz}$, where the LSB of the second setup was used to fill the gap between sidebands of the first setup (and, vice versa, the USB of the first setup fills the IF gap of the second setup) to achieve continuous frequency coverage.

After identification of a $\mathrm{CO}$ line, a seventh frequency setup was used to observe a second $\mathrm{CO}$ line, redshifted to $\sim 75.4 \mathrm{GHz}$, for confirmation. This frequency is almost $10 \mathrm{GHz}$ below the nominal tuning range of CARMA's $3 \mathrm{~mm}$ receivers $(85-116 \mathrm{GHz})$. We thus placed the line in the LSB, using the extended IF range with an IF frequency of $7.5 \mathrm{GHz}$ (same bandwidth as above), and placing the local oscillator (LO) at $82.874 \mathrm{GHz}^{2}$ With this setup, we successfully extended the covered frequency range down to $\sim 74.1 \mathrm{GHz}$, with decreasing sensitivity below $\sim 76 \mathrm{GHz}$. These observations were carried out for three tracks in C configuration between 2010 September 27 and October 10, amounting to a total observing time of $12 \mathrm{hr}$ (6.6 hr on source).

Weather conditions scaled between acceptable and excellent for observations at $3 \mathrm{~mm}$ wavelengths. The nearby quasar J1849+670 was observed every 12-20 minutes for secondary amplitude and phase calibration. The strong calibrator sources $\mathrm{J} 1751+096$, J2015+372, 3C273, 3C345 and 3C454.3 were observed at least once per track for bandpass and secondary flux calibration. Absolute fluxes were bootstrapped relative to Neptune and Mars. Pointing was performed at least every 2-4 hr on nearby sources, using both radio and optical modes. The resulting total calibration is estimated to be accurate within $\sim 15 \%$ (20\% for setup 7).

For data reduction and analysis, the MIRIAD package was used. The final plots were created with the GILDAS package. All data were imaged using "natural" weighting, yielding synthesized beam sizes of 2 ." $8 \times 2$ ". 3 and 4 ". $5 \times 3$ ". 9 at 75.4 and $113.0 \mathrm{GHz}$, and $4 . .5 \times 4^{\prime \prime} .0$ over the full, $41.2 \mathrm{GHz}$ wide bandpass (with a small gap around $80 \mathrm{GHz}$, and excluding line emission). Due to varying effective exposure time, atmospheric conditions and receiver noise, the sensitivity varies somewhat over the full spectral bandpass, in particular toward the edges of the $3 \mathrm{~mm}$ band. The final rms noise values are $24 \mu \mathrm{Jy}$ beam $^{-1}$ over the full bandpass, $0.77 \mathrm{mJy}_{\text {beam }}{ }^{-1}$ over $203 \mathrm{MHz}\left(808 \mathrm{~km} \mathrm{~s}^{-1}\right)$ at $75.4 \mathrm{GHz}$, and $0.52 \mathrm{mJy} \mathrm{beam}^{-1}$ over $292 \mathrm{MHz}\left(773 \mathrm{~km} \mathrm{~s}^{-1}\right)$ at $113.0 \mathrm{GHz}$.

\footnotetext{
2 The USB falls into the frequency range covered by the previous setups.
} 

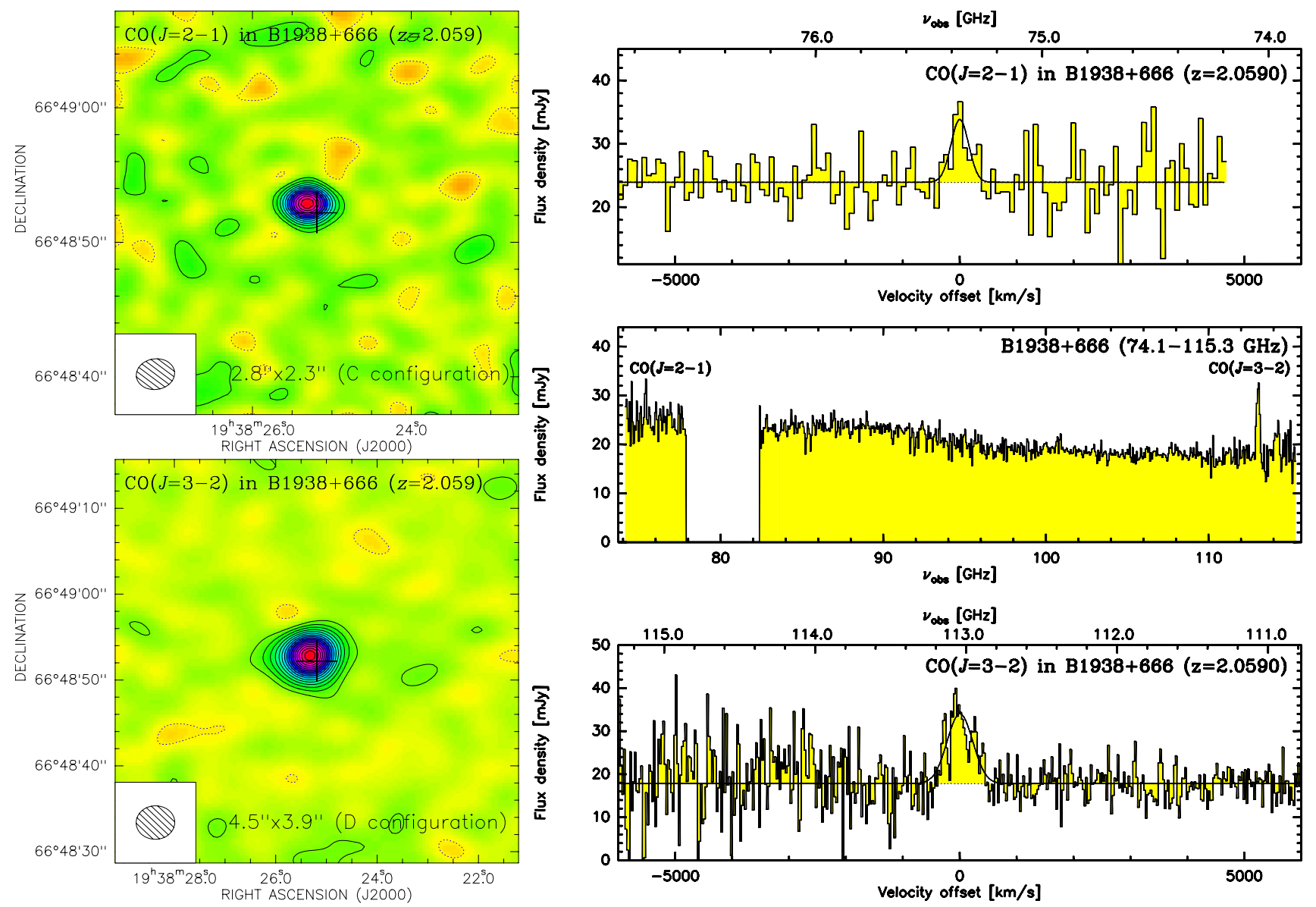

Figure 1. CARMA $\mathrm{CO}(J=2 \rightarrow 1)$ and $\mathrm{CO}(J=3 \rightarrow 2)$ maps (left) and spectra (right) toward $\mathrm{B} 1938+666(z=2.059)$. Contours are plotted in steps of $3 \sigma(1 \sigma=0.77$ and $0.52 \mathrm{mJy}_{\text {beam }}^{-1}$ over 808 and $773 \mathrm{~km} \mathrm{~s}^{-1}$ (203 and $\left.292 \mathrm{MHz}\right)$ ), starting at $\pm 3 \sigma$ (no continuum emission subtracted). The beam sizes are indicated at the bottom of each map. The crosses indicate the (common) pointing center. The middle spectrum (histogram) shows the full covered frequency range of the redshift search from 74.1 to $115.3 \mathrm{GHz}$ at a resolution of $62.496 \mathrm{MHz}$. The top and bottom spectra (histograms) of zoomed-in regions around the $\mathrm{CO}(J=2 \rightarrow 1)$ and $\mathrm{CO}(J=3 \rightarrow 2)$ lines are shown at resolutions of 20.832 and $10.416 \mathrm{MHz}$ ( 83 and $28 \mathrm{~km} \mathrm{~s}^{-1}$ for the $\mathrm{CO} J=2 \rightarrow 1$ and $3 \rightarrow 2$ lines). The solid black curves are Gaussian fits to the spectra. (A color version of this figure is available in the online journal.)

\section{RESULTS}

\section{1. $B 1938+666$}

\subsubsection{Previous Results}

B1938+666 is a radio-selected gravitational lens with a small image separation of only 0.95 in the radio continuum emission associated with its radio-loud AGN (Patnaik et al. 1992). The bright radio continuum emission $(0.577 \pm 0.017 \mathrm{Jy}$ at $1.4 \mathrm{GHz}$ ) is lensed into at least three components that break up into seven subcomponents along a partial Einstein ring, some of which show polarized emission (King et al. 1997). The radio components show a relatively steep spectral index that varies between the different subcomponents $\left(\alpha_{1.612}^{5}=\right.$ $-(1.0-0.5)$ from 1.612 to $5 \mathrm{GHz}$ ), suggesting a superposition of flat and steep spectrum emission associated with lensed core and jet emission. Its $1.6 \mu \mathrm{m}$ continuum emission is lensed into a full, almost perfectly symmetric Einstein ring with 0 '.95 diameter, but only the foreground lensing galaxy is detected at $i$ band and shorter wavelengths (King et al. 1998). This shows that B1938+666 has properties that are clearly different from optically selected, gravitationally lensed quasars. The lensing galaxy has a spectroscopic redshift of $z=0.881$, suggesting $z>1.7$ for B $1938+666$ based on the lensing configuration, with a most likely redshift of $z=2.8$ (Tonry \& Kochanek 2000). Until now, the redshift of B1938+666 was highly uncertain.
In addition to its radio-loud AGN, B1938+666 also hosts a luminous dust bump, with $S_{450 \mu \mathrm{m}}=126 \pm 22 \mathrm{mJy}, S_{850 \mu \mathrm{m}}=$ $34.6 \pm 2.0 \mathrm{mJy}$, and $S_{1.3 \mathrm{~mm}}=14.7 \pm 2.0 \mathrm{mJy}$ (Barvainis \& Ivison 2002). Correcting for synchrotron emission from the AGN, Barvainis \& Ivison (2002) estimate that $32.0 \pm$ $6.7 \mathrm{mJy}$ of the $850 \mu \mathrm{m}$ emission are due to dust heating, likely dominated by star formation. They also find a modelbased lensing magnification factor of $\mu_{\mathrm{L}}=173$. Its extreme submillimeter brightness, dust obscuration in the optical and small Einstein radius (making optical spectroscopic redshift searches unfeasible) make B1938+666 an ideal candidate to attempt a "blind" CO redshift search.

\subsubsection{New Results: "Blind" CO Redshift and Line Emission}

Scanning the whole $3 \mathrm{~mm}$ band from 82.4 to $115.3 \mathrm{GHz}$, we have identified a single CO line in B1938+666 at $113.0425 \mathrm{GHz}$. The lack of detecting a second, lower- $J$ CO line at $82.4<$ $z<113.0 \mathrm{GHz}$ rules out any candidate redshifts at $z>3.0$. Assuming a lower limit of $z=1.7$ as suggested by the lensing configuration, this leaves $\mathrm{CO}(J=3 \rightarrow 2)$ emission at $z=2.059$ as the only likely explanation for the detected line emission. We confirmed this conclusion through a subsequent detection of $\mathrm{CO}(J=2 \rightarrow 1)$ emission at $75.3638 \mathrm{GHz}$.

In Figure 1, the full covered spectral range is shown, along with higher spectral resolution, zoomed-in spectra of the 
Table 2

Observed Line Parameters

\begin{tabular}{|c|c|c|c|c|c|c|}
\hline Target & Line & $z_{\mathrm{CO}}$ & $\begin{array}{c}S_{v} \\
(\mathrm{mJy})\end{array}$ & $\begin{array}{c}\Delta v_{\mathrm{FWHM}} \\
\left(\mathrm{km} \mathrm{s}^{-1}\right)\end{array}$ & $\begin{array}{c}I_{\mathrm{CO}} \\
\left(\mathrm{Jy} \mathrm{km} \mathrm{s}^{-1}\right)\end{array}$ & $r_{J+1, J^{\mathrm{a}}}$ \\
\hline \multirow[t]{7}{*}{ B1938+666 } & $\mathrm{CO}(J=2 \rightarrow 1)$ & $2.0590 \pm 0.0003$ & $9.9 \pm 2.6$ & $366 \pm 124$ & $3.8 \pm 1.1$ & $1.0 \pm 0.3$ \\
\hline & $\mathrm{CO}(J=3 \rightarrow 2)$ & & $16.3 \pm 1.8$ & $529 \pm 75$ & $9.1 \pm 1.1$ & \\
\hline & $\operatorname{HCN}(J=3 \rightarrow 2)$ & & $(3.1 \pm 1.1)$ & $(496 \pm 192)^{b}$ & $(1.62 \pm 0.54)^{\mathrm{c}}$ & \\
\hline & $\mathrm{HCO}^{+}(J=3 \rightarrow 2)$ & & $(1.6 \pm 1.1)$ & $(496 \pm 192)^{b}$ & $(0.86 \pm 0.45)^{\mathrm{c}}$ & \\
\hline & $\mathrm{HNC}(J=3 \rightarrow 2)$ & & $(1.9 \pm 1.1)$ & $(496 \pm 192)^{b}$ & $(1.00 \pm 0.46)^{\mathrm{c}}$ & \\
\hline & $\mathrm{C}_{2} \mathrm{H}(N=3 \rightarrow 2)$ & & $(1.7 \pm 1.1)$ & $(496 \pm 192)^{\mathrm{b}}$ & $(0.89 \pm 0.46)^{\mathrm{c}}$ & \\
\hline & $\mathrm{CN}(N=3 \rightarrow 2)$ & & $(1.5 \pm 1.1)$ & & $(1.0 \pm 0.7)^{\mathrm{c}}$ & \\
\hline HE $0230-2130$ & $\mathrm{CO}(J=3 \rightarrow 2)$ & $2.1664 \pm 0.0005$ & $11.1 \pm 1.5$ & $705 \pm 123$ & $8.3 \pm 1.2$ & \\
\hline HE $1104-1805$ & $\mathrm{CO}(J=3 \rightarrow 2)$ & $2.3221 \pm 0.0004$ & $16.1 \pm 2.4$ & $441 \pm 81$ & $7.5 \pm 1.2$ & \\
\hline \multirow[t]{2}{*}{ B1359+154 } & $\mathrm{CO}(J=3 \rightarrow 2)$ & $3.2399 \pm 0.0003$ & $5.6 \pm 1.7$ & $198 \pm 92$ & $1.2 \pm 0.4$ & $1.2 \pm 0.5$ \\
\hline & $\mathrm{CO}(J=4 \rightarrow 3)$ & & $10.0 \pm 1.6$ & $237 \pm 47$ & $2.5 \pm 0.4$ & \\
\hline
\end{tabular}

Notes.

${ }^{\text {a }}$ Line brightness temperature ratio $\mathrm{CO}(J=n+1 \rightarrow n) / \mathrm{CO}(J=n \rightarrow n-1)$, where $n=2 / 3$ ( $r=1$ : thermalized).

${ }^{\mathrm{b}}$ Fixed to a common line width. The width of the $\mathrm{C}_{2} \mathrm{H}$ line is corrected for broadening due to hyperfine structure.

${ }^{\mathrm{c}}$ Considered not detected. Corresponds to $3 \sigma$ upper limits of $1.6(\mathrm{HCN}), 1.4\left(\mathrm{HCO}^{+}, \mathrm{HNC}, \mathrm{C}_{2} \mathrm{H}\right)$, and $2.1 \mathrm{Jy} \mathrm{km} \mathrm{s}^{-1}(\mathrm{CN})$.

Table 3

Luminosities, Gas Masses and Star Formation Rates (SFRs)

\begin{tabular}{lcclcrrrr}
\hline \hline Source & $\begin{array}{c}D_{\mathrm{L}} \\
(\mathrm{Gpc})\end{array}$ & $\mu_{\mathrm{L}}^{\mathrm{a}}$ & $\begin{array}{c}L_{\mathrm{FIR}} \mathrm{b}, \mathrm{c} \\
\left.\left(10^{12}\right) L_{\odot}\right)\end{array}$ & $\begin{array}{c}L_{\mathrm{CO}}^{\prime} \mathrm{b}, \mathrm{d} \\
\left(10^{10} \mathrm{~K} \mathrm{~km} \mathrm{~s}^{-1} \mathrm{pc}^{2}\right)\end{array}$ & $\begin{array}{c}L_{\mathrm{FIR}} / L_{\mathrm{CO}}^{\prime} \\
\left(L_{\odot}\left(\mathrm{K} \mathrm{km} \mathrm{s}^{-1} \mathrm{pc}^{2}\right)^{-1}\right)\end{array}$ & $\begin{array}{c}M_{\mathrm{gas}}\left(\mathrm{H}_{2}\right)^{\mathrm{e}} \\
\left(10^{10} M_{\odot}\right)\end{array}$ & $\begin{array}{c}\mathrm{SFR}^{\mathrm{f}} \\
\left(M_{\odot} \mathrm{yr}^{-1}\right)\end{array}$ & $\begin{array}{c}\tau_{\mathrm{dep}} \mathrm{g} \\
\left(\mathrm{Myr}^{2}\right)\end{array}$ \\
\hline B1938+666 & 16.31 & 173 & $33 / 0.19$ & $(22.0 \pm 2.5) / 0.13$ & 150 & 0.10 & 30 \\
HE 0230-2130 & 17.36 & 14.5 & $21 / 1.5$ & $(21.9 \pm 3.1) / 1.5$ & 100 & 1.2 & 220 \\
HE 1104-1805 & 18.91 & 10.8 & $16 / 1.5$ & $(22.4 \pm 3.4) / 2.1$ & 70 & 1.7 & 220 \\
B1359+154 & 28.36 & 118 & $11 / 0.093$ & $(7.7 \pm 1.2) / 0.065$ & 140 & 0.052 & 14 \\
\hline
\end{tabular}

Notes.

${ }^{a}$ Adopted from Barvainis \& Ivison 2002.

b Apparent luminosities (not corrected for lensing)/intrinsic luminosities (lensing-corrected).

${ }^{\mathrm{c}} L_{\mathrm{FIR}}$ calculated assuming similar SED shapes as for the Cloverleaf quasar (Weiß et al. 2003).

d Extrapolated to $\mathrm{CO}(J=1 \rightarrow 0)$ luminosity by correcting for excitation, based on excitation modeling of high- $z$ quasars (Riechers et al. 2006, 2009a). Corrections applied: $0 \%$ for $\mathrm{CO}(J=2 \rightarrow 1), 2 \%$ for $\mathrm{CO}(J=3 \rightarrow 2), 6 \%$ for $\mathrm{CO}(J=4 \rightarrow 3)$.

${ }^{\mathrm{e}}$ Assuming a conversion factor of $\alpha=0.8 M_{\odot}\left(\mathrm{K} \mathrm{km} \mathrm{s}^{-1} \mathrm{pc}^{2}\right)^{-1}$ from $L_{\mathrm{CO}(1-0)}^{\prime}$ to $M_{\mathrm{gas}}\left(\mathrm{H}_{2}\right)$ as appropriate for ULIRGs (see Downes \& Solomon 1998).

${ }^{\mathrm{f}}$ Assuming Kennicutt (1998a, 1998b): $\mathrm{SFR}\left[M_{\odot} \mathrm{yr}^{-1}\right]=1.5 \times 10^{-10} L_{\mathrm{FIR}}\left[L_{\odot}\right]$, i.e., $\delta_{\mathrm{MF}} \delta_{\mathrm{SB}}=1.5$ following the notation of Omont et al. (2001): $\delta_{\mathrm{MF}}$ describes the dependence on the mass function of the stellar population, $\delta_{\mathrm{SB}}$ gives the fraction of $L_{\mathrm{FIR}}$ that is actually powered by the starburst and not the AGN.

${ }^{\mathrm{g}}$ Gas depletion timescales, defined as $\tau_{\text {dep }}=M_{\text {gas }} / \mathrm{SFR}$.

emission lines, and emission line maps (continuum not subtracted). As both redshifted $\mathrm{CO}$ lines are close to the edges of the $3 \mathrm{~mm}$ band, the noise across the zoomed-in spectra is not flat and substantially increases below $\sim 75 \mathrm{GHz}$ and above $\sim 114 \mathrm{GHz}$. From Gaussian fitting to the line profiles, we derive line peak flux densities of $S_{v}=9.9 \pm 2.6$ and $16.3 \pm$ $2.8 \mathrm{mJy}$ at FWHM widths of $\Delta v_{\mathrm{FWHM}}=366 \pm 124$ and 529 $\pm 75 \mathrm{~km} \mathrm{~s}^{-1}$ for the $\mathrm{CO}(J=2 \rightarrow 1)$ and $\mathrm{CO}(J=3 \rightarrow 2)$ lines, respectively (see Table 2 ). The line widths are consistent within the errors. A comparison of the line profiles may suggest a more complex structure than a single Gaussian, but more sensitive observations are required to investigate this in more detail. The observed-frame peak velocities correspond to a median redshift of $z_{\mathrm{CO}}=2.0590 \pm 0.0003$. We find velocity-integrated $\mathrm{CO}(J=$ $2 \rightarrow 1)$ and $\mathrm{CO}(J=3 \rightarrow 2)$ line fluxes of $I_{\mathrm{CO}}=3.8 \pm 1.1$ and 9.1 $\pm 1.1 \mathrm{Jy} \mathrm{km} \mathrm{s}^{-1}$. The line brightness temperature ratio of $r_{32}=$ $1.0 \pm 0.3$ is consistent with optically thick, thermalized emission within the errors, consistent with what is found in other high- $z$ quasars (e.g., Riechers et al. 2006).

We derive a lensing-corrected $\mathrm{CO}$ line luminosity of $L_{\mathrm{CO}}^{\prime}=$ $1.3 \times 10^{9}\left(\mu_{\mathrm{L}} / 173\right)^{-1} \mathrm{~K} \mathrm{~km} \mathrm{~s}^{-1} \mathrm{pc}^{2}$. Assuming a conversion factor of $\alpha_{\mathrm{CO}}=0.8 M_{\odot}\left(\mathrm{K} \mathrm{km} \mathrm{s}^{-1} \mathrm{pc}^{2}\right)^{-1}$ from $L_{\mathrm{CO}}^{\prime}$ to gas mass for ultraluminous infrared galaxies (ULIRGs; Downes \& Solomon 1998), we find $M_{\text {gas }}=1.0 \times 10^{9}\left(\mu_{\mathrm{L}} / 173\right)^{-1}\left(\alpha_{\mathrm{CO}} /\right.$
$0.8) M_{\odot}$ (see Table 3 ). In their analysis, Barvainis \& Ivison (2002) introduce a cutoff in $\mu_{\mathrm{L}}$ of a factor of 20 , which may be more appropriate if the gas reservoir extends over a $\gg 100$ pc size region. Under this assumption, $L_{\mathrm{CO}}^{\prime}$ and $M_{\text {gas }}$ would be $\sim 8 \times$ higher, but still at the low end of observed values at high $z$.

\subsubsection{New Results: Constraints on $\mathrm{HCN}, \mathrm{HCO}^{+}, \mathrm{HNC}, \mathrm{C}_{2} \mathrm{H}$, and $\mathrm{CN}$ Line Emission}

Based on the firm CO redshift, we went back to the spectrum to search for emission from dense molecular gas tracers. Fixing the redshift to $z=2.0590$ and all four lines to a common width (the width of the $\mathrm{C}_{2} \mathrm{H}$ line was corrected up by $2.6 \%$ to account for the hyperfine structure; see Figure 2; and Riechers et al. 2007b, 2009a, for more details on the fitting procedure), we simultaneously fitted Gaussian line profiles at the redshifted frequencies of $\mathrm{HCN}(J=3 \rightarrow 2), \mathrm{HCO}^{+}(J=3 \rightarrow 2)$, $\mathrm{HNC}(J=3 \rightarrow 2)$, and $\mathrm{C}_{2} \mathrm{H}(N=3 \rightarrow 2)$ emission, ${ }^{3}$ adding one free parameter to fit the underlying continuum (see Figure 3 ). We

\footnotetext{
3 The rest frequencies of the $\mathrm{HCN}, \mathrm{HCO}^{+}$, and $\mathrm{HNC}(J=3 \rightarrow 2)$ lines are $v_{\text {rest }}=265.886180,267.557619$, and $271.981142 \mathrm{GHz}$. The $\mathrm{C}_{2} \mathrm{H}(N=3 \rightarrow 2)$ line has six spectrally distinguishable hyperfine components at $v_{\text {rest }}=$ 262.004227-262.208439 GHz (see Figure 2).
} 

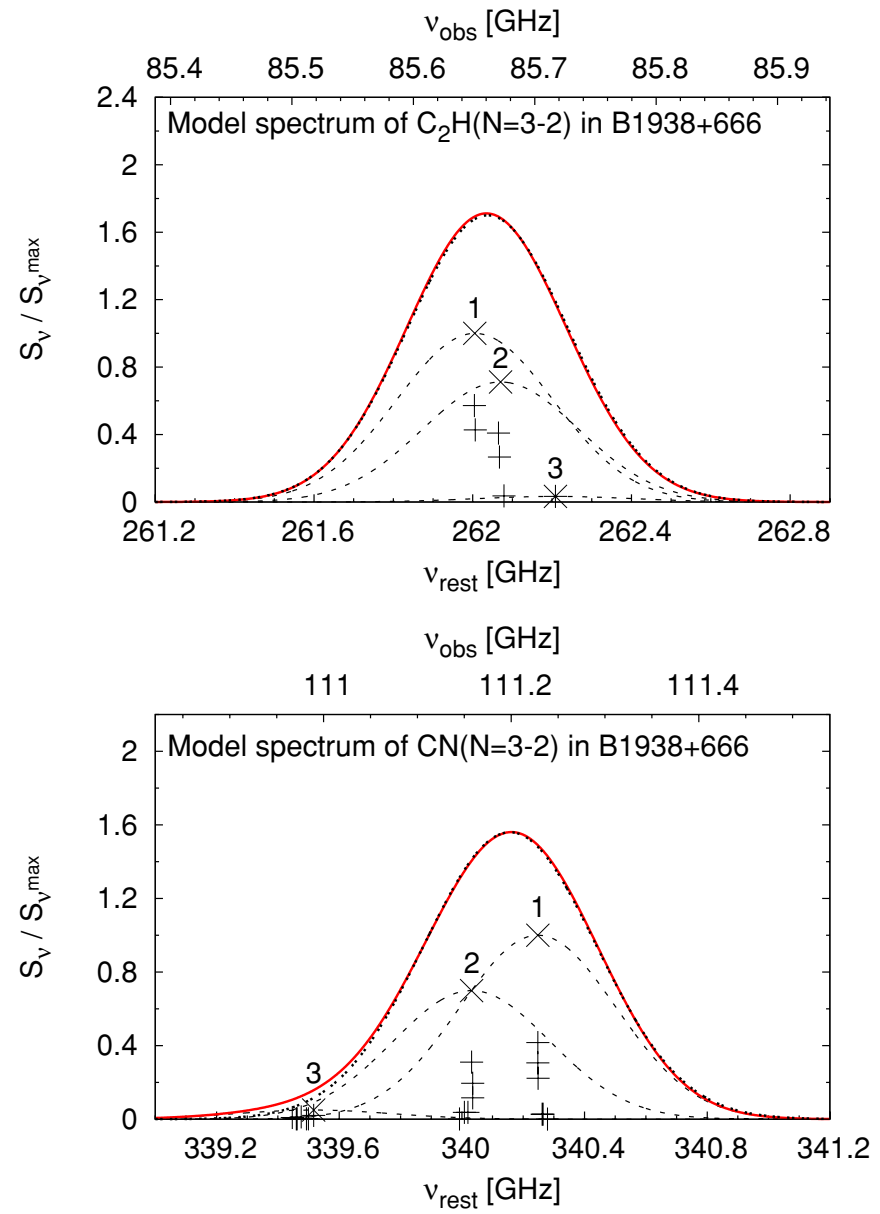

Figure 2. Model spectra of the $\mathrm{C}_{2} \mathrm{H}(N=3 \rightarrow 2)$ and $\mathrm{CN}(N=3 \rightarrow 2)$ emission toward B1938+666. The relative intensities are computed in local thermodynamic equilibrium (see Riechers et al. 2007b, 2009a, for details on the computation). The plus signs indicate all hyperfine components, for each of which the same line width as for the $\operatorname{CO}(J=3 \rightarrow 2)$ line is assumed. The crosses and dashed lines indicate the peak intensities and profiles of the three main subcomponents (labeled 1-3), summed over hyperfine components close in frequency. The vertical axes indicate the predicted intensity, normalized to the brightest subcomponent. The two brightest of these subcomponents for each line are indicated in Figure 3. The solid lines are the profiles summed over all components. The thick dotted line indicates single Gaussian profiles fitted to the summed profiles and have widths that are by $\sim 2.6 \%\left(\mathrm{C}_{2} \mathrm{H}\right)$ and $\sim 10 \%(\mathrm{CN})$ larger than that the individual components (and are used to fit the observations to minimize the number of fitting parameters).

(A color version of this figure is available in the online journal.)

find $S_{v}=3.1 \pm 1.1,1.6 \pm 1.1,1.9 \pm 1.1$, and $1.7 \pm 1.1 \mathrm{mJy}$ at a common $\Delta v_{\mathrm{FWHM}}=496 \pm 192 \mathrm{~km} \mathrm{~s}^{-1}$ for the $\mathrm{HCN}, \mathrm{HCO}^{+}$, and $\mathrm{HNC}(J=3 \rightarrow 2)$ and $\mathrm{C}_{2} \mathrm{H}(N=3 \rightarrow 2)$ lines, respectively. This corresponds to $I_{\mathrm{HCN}}=1.62 \pm 0.54, I_{\mathrm{HCO}^{+}}=0.86 \pm 0.45, I_{\mathrm{HNC}}=$ $1.00 \pm 0.46$, and $I_{\mathrm{C}_{2} \mathrm{H}}=0.89 \pm 0.46 \mathrm{Jy} \mathrm{km} \mathrm{s}^{-1}$, respectively. We also searched for $\mathrm{CN}$ emission, simultaneously fitting the $\mathrm{CN}(N=3 \rightarrow 2)$ and $\mathrm{CO}(J=3 \rightarrow 2)$ lines and the underlying continuum emission (see Figure 3 ). The width of the $\mathrm{CN}$ line was corrected up by $10 \%$ to account for the hyperfine structure (see Figure 2). ${ }^{4}$ The fit yields $S_{v}=1.5 \pm 1.1 \mathrm{mJy}$. corresponding to $I_{\mathrm{HCN}}=1.0 \pm 0.7 \mathrm{Jy} \mathrm{km} \mathrm{s}^{-1}$.

The $\operatorname{HCN}(J=3 \rightarrow 2)$ line is marginally detected at $3.0 \sigma$ level, but we conservatively treat it as an upper limit in the following. The other four lines yield 1.4-2.2 $\sigma$ signals, and thus are not

\footnotetext{
4 The $\mathrm{CN}(N=3 \rightarrow 2)$ line has 19 spectrally distinguishable hyperfine
} components at $v_{\text {rest }}=339.446777-340.279166 \mathrm{GHz}$ (see Figure 2). detected. Translating their fluxes to $3 \sigma$ upper limits yields $I<$ $1.4\left(\mathrm{HCO}^{+}, \mathrm{HNC}, \mathrm{C}_{2} \mathrm{H}\right)$ and $<2.1 \mathrm{Jy} \mathrm{km} \mathrm{s}^{-1}(\mathrm{CN})$. Given that the simultaneously fitted $\mathrm{HCN}, \mathrm{HCO}^{+}, \mathrm{HNC}$, and $\mathrm{C}_{2} \mathrm{H}$ lines all yield positive signals at 1.9-3.0 $\sigma$ level, we attempted to stack their spectra. This yields a weighted average flux of $1.05 \pm$ $0.24 \mathrm{Jy} \mathrm{km} \mathrm{s}^{-1}$, corresponding to a signal of $4.4 \sigma$ significance. This stacked signal corresponds to $19 \% \pm 5 \%$ of the $\operatorname{CO}(J=$ $3 \rightarrow 2$ ) luminosity (i.e., not corrected for line excitation). This is consistent with the median $\mathrm{HCN} / \mathrm{CO} L^{\prime}$ ratios (a measure of the dense gas fraction in galaxies) in nearby ULIRGs and $z>2$ quasars (e.g., Gao et al. 2007; Riechers et al. 2007a).

\subsubsection{New Results: Continuum Emission}

As shown in Figure 1, we detect continuum emission toward B1938+666 over the entire observed wavelength range. Simultaneous fits to the line and continuum emission suggest continuum strengths of $23.89 \pm 0.32$ and $17.76 \pm 0.26$ mJy below the $\mathrm{CO}(J=2 \rightarrow 1)$ and $\mathrm{CO}(J=3 \rightarrow 2)$ lines, and $23.15 \pm 0.15 \mathrm{mJy}$ below the $\mathrm{HCN}(J=3 \rightarrow 2), \mathrm{HCO}^{+}(J=3 \rightarrow 2), \operatorname{HNC}(J=3 \rightarrow 2)$, and $\mathrm{C}_{2} \mathrm{H}(N=3 \rightarrow 2)$ lines. The strength and slope of the emission are consistent with synchrotron emission from the radio-loud AGN. The spectrum may suggest a more complex continuum slope than a simple power law. However, due to the variability of the phase calibrator over the four month course of the observations, there is a residual uncertainty in the flux calibration between the different frequency settings. We thus conclude that the continuum slope is consistent with a power law within the errors. By averaging the line-free channels over the entire observed frequency range and fitting a two-dimensional Gaussian to the $u-v$ data, we find a $3.1 \mathrm{~mm}$ continuum flux of $20.84 \pm$ $0.14 \mathrm{mJy}$. In Figure 4, a map of the averaged continuum emission is shown. From the fit, we also find a continuum size of $\left(1^{\prime \prime} .48 \pm 0^{\prime \prime} .05\right) \times\left(1^{\prime \prime} .10 \pm 0^{\prime \prime} .05\right)$ at a position angle of $85^{\circ} \pm 5^{\circ}$. Accounting for interferometric seeing (which is not part of the above errors), this is consistent with the 0.95 diameter of the Einstein ring, and the continuum emission to be dominated by the radio-loud AGN.

\subsubsection{Origin of the CO/Continuum Emission}

In Figure 5, an overlay of the $\mathrm{CO}(J=3 \rightarrow 2)$ and underlying continuum emission on top of observed-frame $1.6 \mu \mathrm{m}$ continuum emission in B1938+666 is shown (Hubble Space Telescope, HST, image from King et al. 1998; all HST images are data products from the Hubble Legacy Archive). ${ }^{5}$ The peak of the CO/ continuum emission is consistent with the brightest rest-frame optical $(523 \mathrm{~nm})$ emission region along the Einstein ring. This suggests that the optical and (rest-frame) (sub)millimeter emission (observed frame $2.6-4.0 \mathrm{~mm}$ ) are likely cospatial, with the optical emission being dominated by stellar light above the Balmer break. The lack of point-like images along the Einstein ring suggests that the radio-loud AGN is optically obscured (or, alternatively, has low optical luminosity). The strength and spectral slope of the rest-frame submillimeter continuum emission make it unlikely that the AGN is much less magnified than its host galaxy. Thus, B1938+666 likely hosts a type-2 AGN along with its starburst.

\subsubsection{Absorption Line Searches}

Given the high $3 \mathrm{~mm}$ continuum flux of B1938+666, we have searched the spectrum for absorption lines from foreground

\footnotetext{
5 http://hla.stsci.edu/
} 

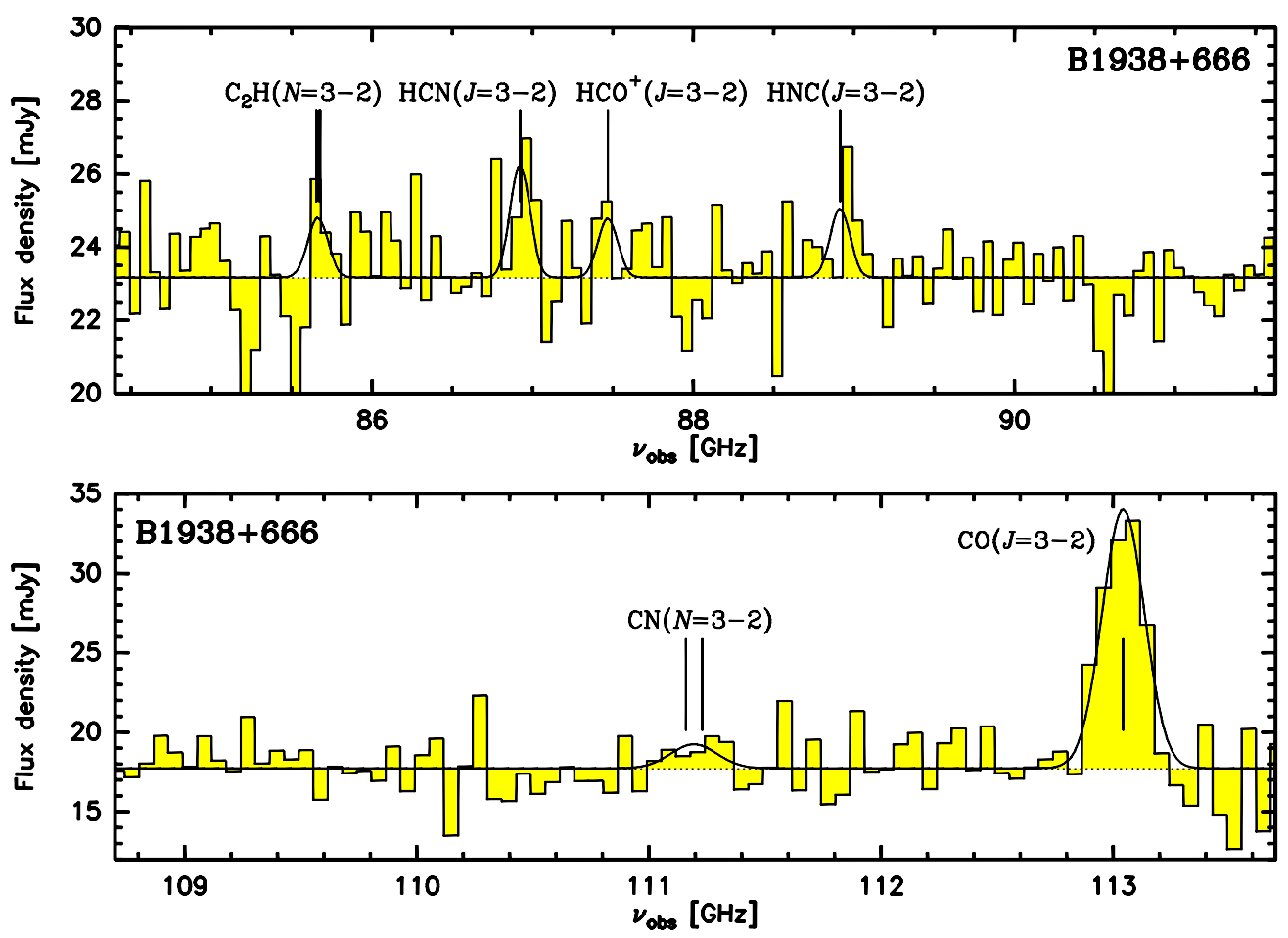

Figure 3. Search for $\mathrm{HCN}(J=3 \rightarrow 2), \mathrm{HCO}^{+}(J=3 \rightarrow 2), \mathrm{HNC}(J=3 \rightarrow 2), \mathrm{C}_{2} \mathrm{H}(N=3 \rightarrow 2)$, and $\mathrm{CN}(N=3 \rightarrow 2)$ emission toward $\mathrm{B} 1938+666(z=2.059)$. The spectra (histogram) are shown at the same resolution as in the middle panel of Figure 1. Top: the solid black curve shows a simultaneous Gaussian fit to all four lines with a common line width (corrected for the $\mathrm{C}_{2} \mathrm{H}$ hyperfine structure; see Figure 2) and the underlying continuum emission, with the redshift fixed to $z=2.0590$. The peak frequencies of the $\mathrm{HCN}(J=3 \rightarrow 2), \mathrm{HCO}^{+}(J=3 \rightarrow 2)$, and $\mathrm{HNC}(J=3 \rightarrow 2)$ lines as well as the main hyperfine structure components of the $\mathrm{C}_{2} \mathrm{H}(N=3 \rightarrow 2)$ line are indicated by the vertical lines. Bottom: the solid black curve shows a simultaneous Gaussian fit to both lines with a common line width (corrected for the $\mathrm{CN}$ hyperfine structure; see Figure 2) and the underlying continuum emission. The peak frequencies of the main hyperfine components of $\mathrm{CN}(N=3 \rightarrow 2)$ and that of the $\mathrm{CO}(J=$ $3 \rightarrow 2$ ) line are indicated by the vertical lines.

(A color version of this figure is available in the online journal.)
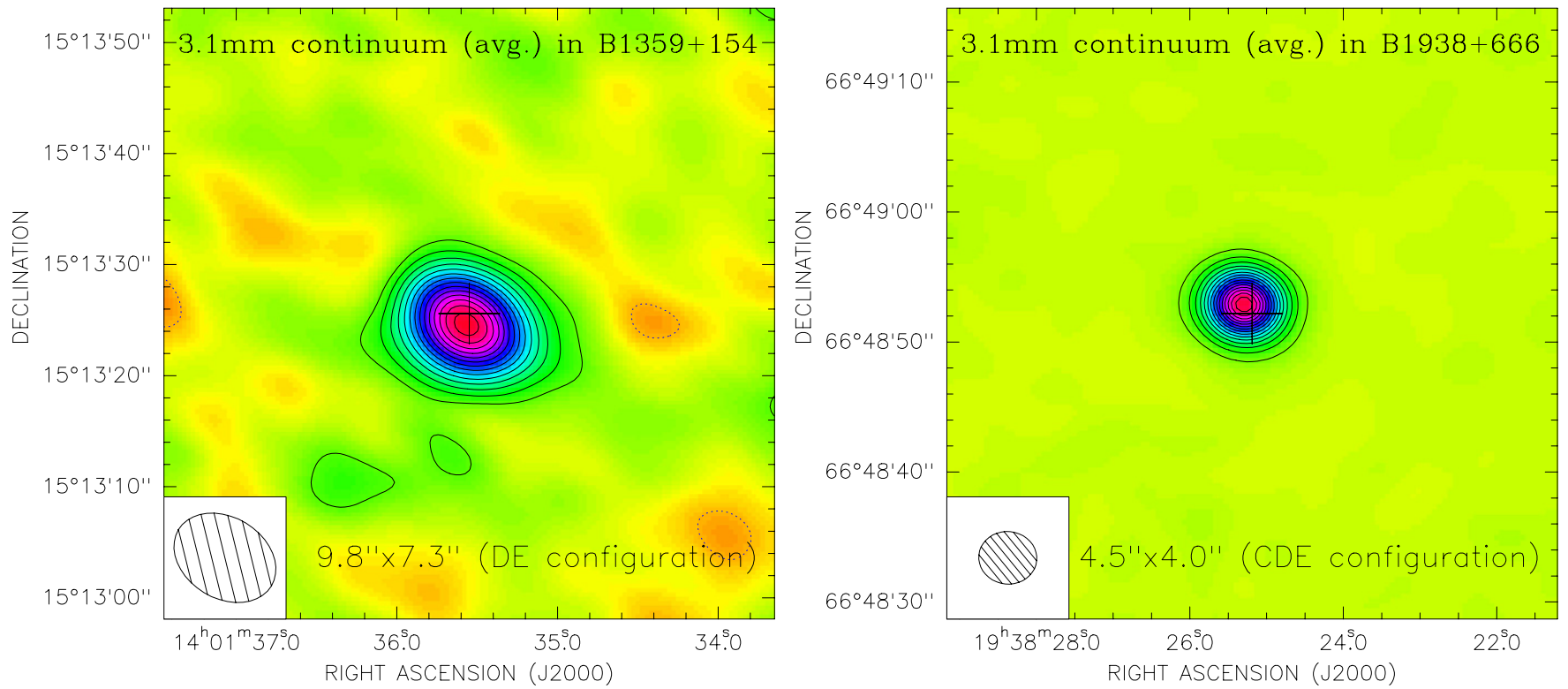

Figure 4. $3.1 \mathrm{~mm}$ continuum emission in B1359+154 and B1938+666, averaged over all line-free data in the lower and upper sidebands. The beam sizes are indicated at the bottom. The crosses indicate the same position as in Figures 1 and 7 . Left: contours are plotted in steps of $2 \sigma\left(1 \sigma=0.12 \mathrm{mJy}^{\circ}\right.$ beam $\left.{ }^{-1}\right)$, starting at $\pm 3 \sigma$. Right: contours are plotted in steps of $50 \sigma\left(1 \sigma=24 \mu \mathrm{Jy}_{\text {beam }}{ }^{-1}\right)$, starting at $\pm 50 \sigma$.

(A color version of this figure is available in the online journal.)

sources. In Figure 6, three spectral regions are shown that cover the absorption line frequencies of $\mathrm{HCN}(J=1 \leftarrow 0), \mathrm{HCO}^{+}(J=$ $1 \leftarrow 0)$, and $\mathrm{HNC}(J=1 \leftarrow 0)$ in the Milky Way (i.e., at $z=$ $0)$, and of $\operatorname{HCN}(J=2 \leftarrow 1), \mathrm{HCO}^{+}(J=2 \leftarrow 1)$, and $\operatorname{HNC}(J=$ $2 \leftarrow 1)$ at $z=0.881$, the redshift of the lensing galaxy. HCN,
$\mathrm{HCO}^{+}$, or $\mathrm{HNC}$ are abundant molecules typically found in dense regions, and typically produce some of the deepest absorption features (e.g., Wiklind \& Combes 1995, 1996). The spectra are normalized to an arbitrary flux scale, and re-binned to $10.416 \mathrm{MHz}$ ( 35 and $33 \mathrm{~km} \mathrm{~s}^{-1}$ ), to enable searches for narrow 

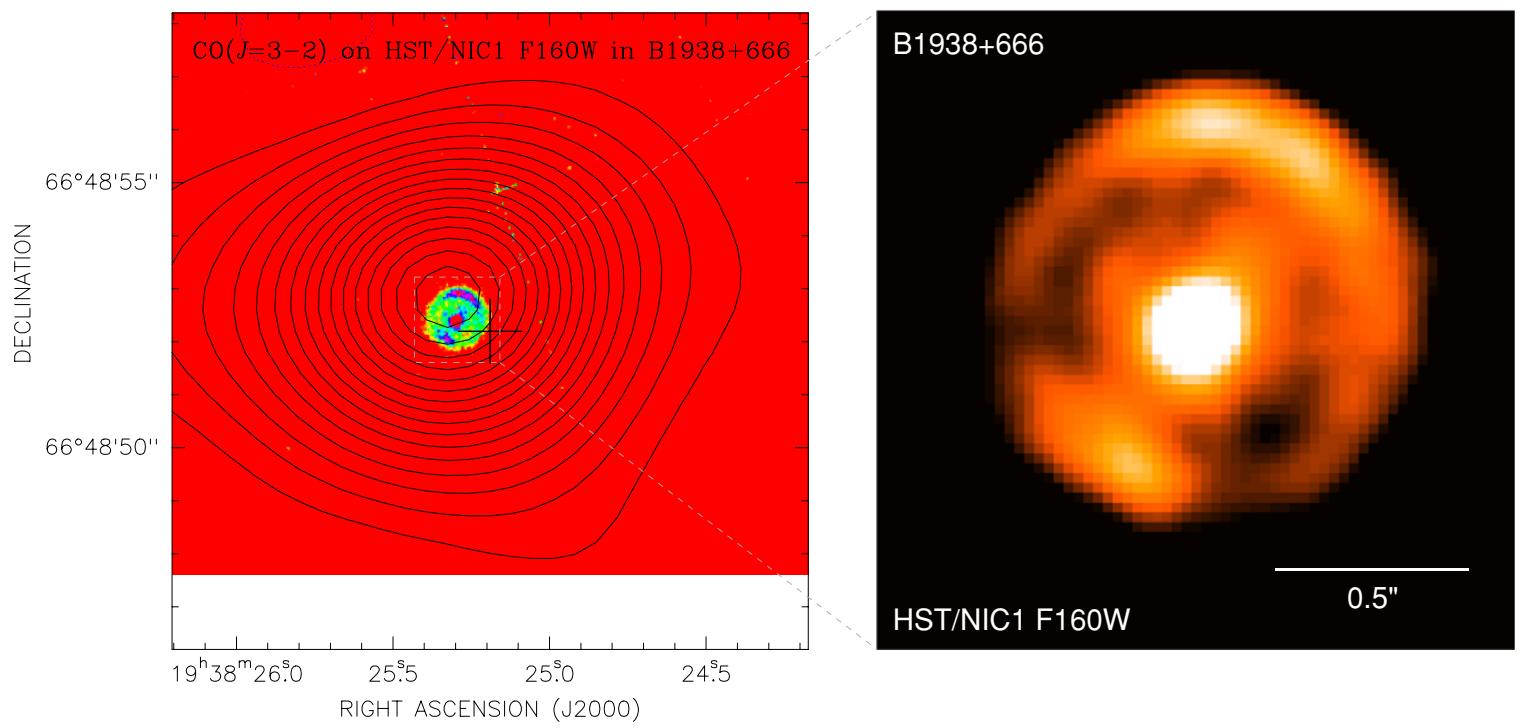

Figure 5. Overlay of $\mathrm{CO}(J=3 \rightarrow 2)$ (contours) and $1.6 \mu \mathrm{m}$ (rest-frame $523 \mathrm{~nm}$ ) continuum emission (left: $H S T / \mathrm{NICMOS1}$; image not cleaned; the background source is not detected at 555 and $814 \mathrm{~nm}$ ), and zoomed-in intensity map of the $1.6 \mu \mathrm{m}$ emission (right: smoothed with a $0^{\prime \prime} 1$ Gaussian kernel) in B1938+666. The cross indicates the same position as in Figure 1. The source is lensed into a full Einstein ring, and the central spot is the foreground lensing galaxy.

(A color version of this figure is available in the online journal.)
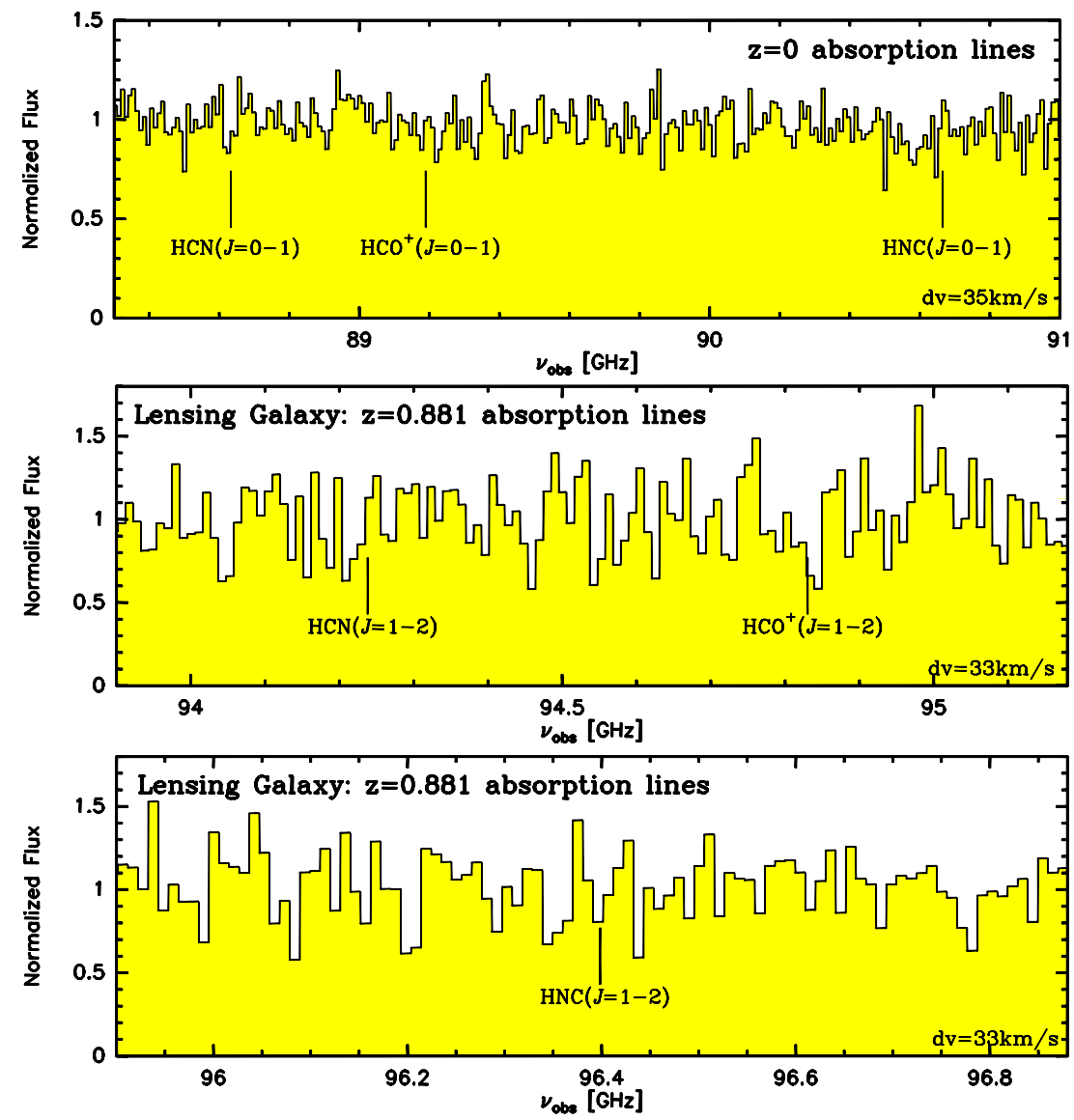

Figure 6. Absorption line searches toward the continuum of B1938+666. The top panel shows a frequency region covering the $\mathrm{HCN}(J=1 \leftarrow 0), \mathrm{HCO}^{+}(J=1 \leftarrow 0)$, and $\mathrm{HNC}(J=1 \leftarrow 0)$ lines at $z=0$ (i.e., Galactic regions) at a velocity resolution of $35 \mathrm{~km} \mathrm{~s}^{-1}(10.416 \mathrm{MHz})$. The middle and bottom panels show frequency regions covering the $\mathrm{HCN}(J=2 \leftarrow 1), \mathrm{HCO}^{+}(J=2 \leftarrow 1)$, and $\mathrm{HNC}(J=2 \leftarrow 1)$ lines at $z=0.881$, i.e., the redshift of the lensing galaxy, at a velocity resolution of $33 \mathrm{~km} \mathrm{~s}{ }^{-1}$ $(10.416 \mathrm{MHz})$. All spectra are normalized to an arbitrary flux scale.

(A color version of this figure is available in the online journal.)

lines from compact molecular structures. At this resolution, the continuum emission is detected in every single velocity channel, but at a relatively moderate signal-to-noise ratio. We do not detect any absorption lines. Assuming a velocity width that is at least comparable to the resolution of these spectra, this suggests that $<20 \%$ of the continuum emission of B1938+666 
are absorbed by Galactic or nearby extragalactic foreground structures and $<40 \%$ are absorbed by the molecular gas $(\mathrm{HCN}$, $\mathrm{HCO}^{+}$, or $\mathrm{HNC}$ ) in the lensing galaxy.

\subsection{HE 0230-2130: the Southern Cloverleaf}

\subsubsection{Previous Results}

HE 0230-2130 is an optically selected, quadruply lensed quasar at an optical redshift of $z_{\text {opt }}=2.163 \pm 0.003$ with a maximum image separation of 2 '.15 (Wisotzki et al. 1999; Anguita et al. 2008), similar to the well-known Cloverleaf quasar (Magain et al. 1988). It is lensed by two galaxies at almost identical redshift $\left(z_{\mathrm{G} 1}=0.523 \pm 0.001, z_{\mathrm{G} 2}=0.526 \pm 0.002\right.$; Eigenbrod et al. 2006). This "Southern Cloverleaf" is radioquiet, but has a luminous dust bump, with $S_{450 \mu \mathrm{m}}=77 \pm 13 \mathrm{mJy}$ and $S_{850 \mu \mathrm{m}}=21.0 \pm 2.2 \mathrm{mJy}$. These fluxes are $\sim 35 \%$ of those found for the Cloverleaf and consistent with the same spectral slope (Barvainis \& Ivison 2002). Barvainis and Ivison find a model-based lensing magnification factor of $\mu_{\mathrm{L}}=14.5$, which is similar to that of the Cloverleaf $\left(\mu_{\mathrm{L}}=11\right.$; Venturini \& Solomon 2003). HE 0230-2130 also exhibits luminous emission from polycyclic aromatic hydrocarbons (PAHs; Lutz et al. 2008). Together, these properties suggest the presence of a substantial molecular gas reservoir.

\subsubsection{New Results}

We detect luminous, marginally resolved $\mathrm{CO}(J=3 \rightarrow 2)$ emission toward HE 0230-2130 at $>9 \sigma$ significance (Figure 7). From Gaussian fitting to the line profile, we find $S_{v}=11.1 \pm$ $1.5 \mathrm{mJy}$ and $\Delta v_{\text {FWHM }}=705 \pm 123 \mathrm{~km} \mathrm{~s}^{-1}$, corresponding to $I_{\mathrm{CO}}=8.3 \pm 1.2 \mathrm{Jy} \mathrm{km} \mathrm{s}^{-1}$ (see Table 2). We do not detect the underlying continuum down to a $2 \sigma$ limit of $<1.5 \mathrm{mJy}$. The $\mathrm{CO}$ line emission yields a host galaxy redshift of $z_{\mathrm{CO}}=2.1664 \pm$ 0.0005 , which is consistent with the optical redshift of the quasar within the errors $\left(d z=\left\|z_{\mathrm{CO}}-z_{\mathrm{opt}}\right\| \simeq 0.003\right)$.

The CO line emission in HE 0230-2130 appears resolved both spatially and in velocity. There is a clear difference between the CO line width at the peak position (dashed spectrum in Figure 7) and the spatially integrated line width. In Figure 8, a first moment map is shown (clipped below $1 \sigma$ in the individual velocity channels). Despite the moderate signal-to-noise ratio of this map, a clear velocity gradient is apparent. Accounting for beam convolution, the spatial and velocity structure is consistent with the lensed size of the source as defined by the maximum images separation of 2 .' 15 within the uncertainties.

Assuming a $2 \%$ correction for subthermal excitation of the $\mathrm{CO}(J=3 \rightarrow 2)$ line $^{6}$ (based on $\mathrm{CO}$ line radiative transfer modeling of high- $z$ quasars, e.g., Riechers et al. 2006, 2009b), we derive $L_{\mathrm{CO}}^{\prime}=1.5 \times 10^{10}\left(\mu_{\mathrm{L}} / 14.5\right)^{-1} \mathrm{~K} \mathrm{~km} \mathrm{~s}^{-1} \mathrm{pc}^{2}$ and $M_{\text {gas }}=1.2 \times 10^{10}\left(\mu_{\mathrm{L}} / 14.5\right)^{-1}\left(\alpha_{\mathrm{CO}} / 0.8\right) M_{\odot}($ see Table 3$)$.

\subsection{HE 1104-1805}

\subsubsection{Previous Results}

HE 1104-1805 is an optically selected double image quasar with a lens image separation of 3". 19 at an optical redshift of $z_{\text {opt }}=2.3192 \pm 0.0007$ (Wisotzki et al. 1995; Sulentic et al. 2006). It is lensed by a galaxy at $z_{\mathrm{G}}=0.729 \pm 0.001$ (Lidman et al. 2000). Its host galaxy is lensed into an Einstein ring (Peng

\footnotetext{
6 This model-based, small correction factor is not statistically significant, but applied for consistency with estimates based on higher- $J$ lines, as described below. Within the uncertainties of our measurements, the $\mathrm{CO}(J=3 \rightarrow 2)$ lines in our targets are consistent with thermal excitation.
}

et al. 2006). It has a supermassive black hole mass of $M_{\mathrm{BH}}=$ $2.4 \times 10^{9} M_{\odot}$ and follows the relation between black hole mass and rest-frame $R$-band luminosity of the host galaxy $\left(M_{\mathrm{BH}}-L_{\mathrm{R}}\right)$ for $z \gtrsim 1.7$ galaxies (Peng et al. 2006). Estimating its bolometric AGN luminosity from its $M_{\mathrm{BH}}$ and $B$-band magnitude (Peng et al. 2006), HE 1104-1805 follows the $L_{\mathrm{bol}}-L_{\mathrm{FIR}}$ relation for PG quasars and is consistent with the values found for other high- $z$ FIR-luminous quasars within the uncertainties (e.g., Omont et al. 2003; Wang et al. 2008; see Table 3 for $L_{\text {FIR }}$ ). HE 1104-1805 is radio-quiet, but has a luminous dust bump, with $S_{850 \mu \mathrm{m}}=14.8 \pm 3.0 \mathrm{mJy}$ and $S_{1.3 \mathrm{~mm}}=5.3 \pm 0.9 \mathrm{mJy}$. It is magnified by a factor of $\mu_{\mathrm{L}}=10.8$ (Barvainis \& Ivison 2002). It also exhibits luminous PAH emission (Lutz et al. 2008).

\subsubsection{New Results}

We detect luminous, spatially resolved $\mathrm{CO}(J=3 \rightarrow 2)$ emission toward both lens images of HE 1104-1805 at $>8 \sigma$ and $>4 \sigma$ significance (Figure 7). From Gaussian fitting to the line profile, we find $S_{v}=16.1 \pm 2.4 \mathrm{mJy}$ and $\Delta v_{\mathrm{FWHM}}=441 \pm$ $81 \mathrm{~km} \mathrm{~s}^{-1}$, corresponding to $I_{\mathrm{CO}}=7.5 \pm 1.2 \mathrm{Jy} \mathrm{km} \mathrm{s}^{-1}$ (see Table 2). We do not detect the underlying continuum down to a $2 \sigma$ limit of $<1.5 \mathrm{mJy}$. The $\mathrm{CO}$ line emission yields a host galaxy redshift of $z_{\mathrm{CO}}=2.3221 \pm 0.0004$, which is close to the optical redshift of the quasar $\left(d z=\left\|z_{\mathrm{CO}}-z_{\text {opt }}\right\| \simeq 0.003\right)$.

Assuming a $2 \%$ correction for subthermal excitation of the $\mathrm{CO}(J=3 \rightarrow 2)$ line, we derive $L_{\mathrm{CO}}^{\prime}=2.1 \times 10^{10}\left(\mu_{\mathrm{L}} /\right.$ $14.5)^{-1} \mathrm{~K} \mathrm{~km} \mathrm{~s}^{-1} \mathrm{pc}^{2}$ and $M_{\mathrm{gas}}=1.7 \times 10^{10}\left(\mu_{\mathrm{L}} / 10.8\right)^{-1}\left(\alpha_{\mathrm{CO}} /\right.$ 0.8) $M_{\odot}$ (see Table 3$)$.

\subsubsection{Origin of the CO Emission}

In Figure 9, overlays of the $\mathrm{CO}(J=3 \rightarrow 2)$ emission on top of observed-frame $555 \mathrm{~nm}, 814 \mathrm{~nm}$, and $1.6 \mu \mathrm{m}$ continuum emission in HE 1104-1805 are shown (HST images from Remy et al. 1998; Léhar et al. 2000). The CO emission is clearly associated with the two (point-like) images of the AGN. The emission peaks are slightly spatially offset. This may indicate that the AGN does not reside at the center of the molecular gas reservoir. However, this small offset may as well be due to smaller scale dynamics of the molecular gas that are averaged over in the CO emission line map. The CO images are consistent with being resolved along the Einstein radius, but observations at higher spatial resolution and sensitivity are desirable to study the structure and dynamics of the lensed gas reservoir in more detail.

\section{4. $B 1359+154$ \\ 3.4.1. Previous Results}

$\mathrm{B} 1359+154$ is a rare, sextuply lensed, radio-loud quasar at an optical redshift of $z_{\text {opt }}=3.235 \pm 0.002$, with a maximum image separation of 1 .'71 (Myers et al. 1999). It was selected in the radio, but in contrast to B1938+666, is an optically bright quasar. It is lensed by a compact group at $z_{\mathrm{G}} \simeq 1$, with three primary lensing galaxies of similar luminosities, which are situated on the vertices of a triangle, separated by $\sim 0.7$ (photometric/ lensing redshifts are $z_{\mathrm{G} 1}=1.35 \pm 0.16, z_{\mathrm{G} 2}=0.88 \pm 0.06$, and $z_{\mathrm{G} 3}=0.94 \pm 0.07$; Rusin et al. 2001). Very compact (3-9 mas lensed sizes along their major axes), flat spectrum $\left(\alpha_{1.7}^{5} \simeq-0.3\right.$ from 1.7 to $5 \mathrm{GHz}$ ) radio cores are associated with all six images, and the brightest three images show radio jets extending out to tens of mas (lensed) scales (Rusin et al. 2001). B1359+154 has a luminous dust bump, with $S_{450 \mu \mathrm{m}}=39 \pm 10 \mathrm{mJy}$ and 

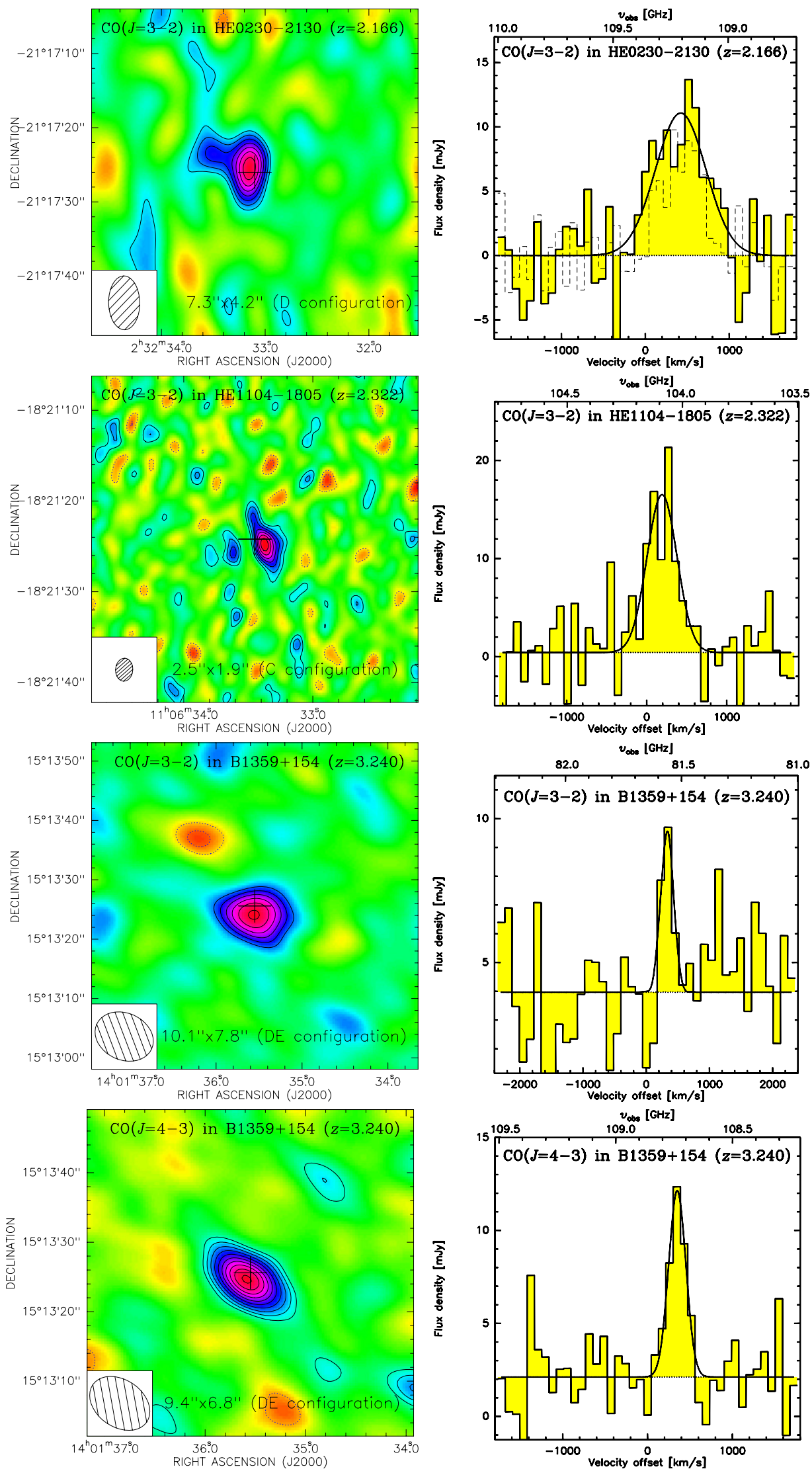

Figure 7. CARMA $\mathrm{CO}(J=3 \rightarrow 2)$ and $\mathrm{CO}(J=4 \rightarrow 3)$ maps (left) and spectra (right) toward HE $0230-2130(z=2.166)$, $\mathrm{HE} 1104-1805(z=2.322)$, and $\mathrm{B} 1359+154$ $(z=3.240)$. Contours are plotted in steps of $1 \sigma\left(0.52,0.65,0.97\right.$, and $0.71 \mathrm{mJy}^{-1} \mathrm{bm}^{-1}$ over $1114,450,344$, and $430 \mathrm{~km} \mathrm{~s}^{-1}(406.25,156.25,93.75$, and $\left.156.25 \mathrm{MHz})\right)$, starting at $\pm 3 \sigma, \pm 2 \sigma, \pm 3 \sigma$, and $\pm 3 \sigma$ (no continuum emission subtracted). The beam sizes are indicated at the bottom of each map. The crosses indicate the pointing centers. The spectra (histograms) are shown at a resolution of $31.25 \mathrm{MHz}\left(86,90,115\right.$, and $\left.86 \mathrm{~km} \mathrm{~s}^{-1}\right)$. The solid black curves are Gaussian fits to the spectra. The dashed histogram in the top right panel indicates the peak position spectrum only.

(A color version of this figure is available in the online journal.) 


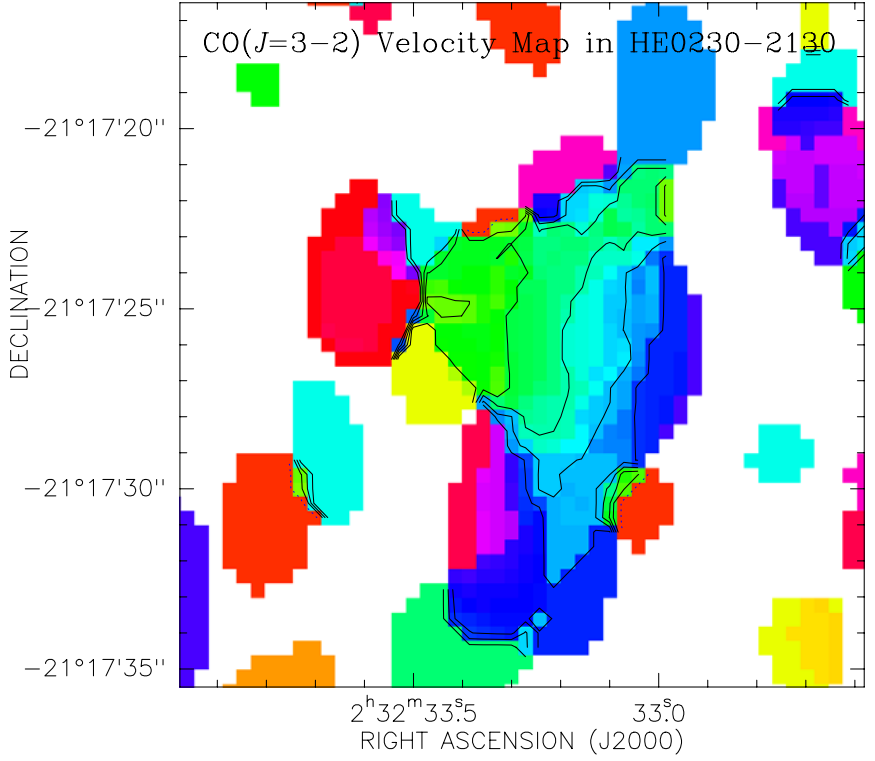

Figure 8. First moment map of the $\mathrm{CO}(J=3 \rightarrow 2)$ emission in HE 0230-2130 shown in Figure 7. Velocity contours are in steps of $\Delta v=100 \mathrm{~km} \mathrm{~s}^{-1}$.

(A color version of this figure is available in the online journal.)

$S_{850 \mu \mathrm{m}}=11.5 \pm 1.9 \mathrm{mJy}$. It is magnified by a factor of $\mu_{\mathrm{L}}=$ 118 (Barvainis \& Ivison 2002).

\subsubsection{New Results: Line Emission}

We detect luminous $\mathrm{CO}(J=3 \rightarrow 2)$ and $\mathrm{CO}(J=4 \rightarrow 3)$ emission toward B1359+154 (Figure 7). From Gaussian fitting to the line profiles, we find $S_{v}=5.6 \pm 1.7$ and $10.0 \pm 1.6 \mathrm{mJy}$, and $\Delta v_{\mathrm{FWHM}}=198 \pm 92$ and $237 \pm 47 \mathrm{~km} \mathrm{~s}^{-1}$ for the $\mathrm{CO}(J=$ $3 \rightarrow 2)$ and $\operatorname{CO}(J=4 \rightarrow 3)$ lines, respectively. The line widths are consistent within the errors. This corresponds to $I_{\mathrm{CO}}=$ $1.2 \pm 0.4$ and $2.5 \pm 0.4 \mathrm{Jy} \mathrm{km} \mathrm{s}^{-1}$ (see Table 2). The CO line emission yields a median host galaxy redshift of $z_{\mathrm{CO}}=3.2399 \pm$ 0.0003 , which is close to the optical redshift of the quasar $(d z=$ $\left.\left\|z_{\mathrm{CO}}-z_{\mathrm{opt}}\right\| \simeq 0.005\right)$. The line brightness temperature ratio of $r_{43}=1.2 \pm 0.5$ is consistent with optically thick, thermalized emission within the errors.

Assuming a $6 \%$ correction for subthermal excitation of the (higher signal-to-noise ratio) $\mathrm{CO}(J=4 \rightarrow 3)$ line, we derive
$L_{\mathrm{CO}}^{\prime}=6.5 \times 10^{8}\left(\mu_{\mathrm{L}} / 118\right)^{-1} \mathrm{~K} \mathrm{~km} \mathrm{~s}^{-1} \mathrm{pc}^{2}$ and $M_{\mathrm{gas}}=5.2 \times$ $10^{8}\left(\mu_{\mathrm{L}} / 118\right)^{-1}\left(\alpha_{\mathrm{CO}} / 0.8\right) M_{\odot}$ (see Table 3$)$, comparable to what is found in the lensed Lyman break galaxy MS 1512-cB58 (Riechers et al. 2010a). Under the assumption of $\mu_{\mathrm{L}}=20, L_{\mathrm{CO}}^{\prime}$ and $M_{\text {gas }}$ would be $\lesssim 6 \times$ higher, but still at the low end of observed values at high- $z$.

\subsubsection{New Results: Continuum Emission}

As shown in Figure 7, we detect continuum emission toward B1359+154 under the CO lines. Simultaneous fits to the line and continuum emission suggest continuum strengths of $3.97 \pm$ 0.35 and $2.11 \pm 0.32 \mathrm{mJy}$ below the $\operatorname{CO}(J=3 \rightarrow 2)$ and $\mathrm{CO}(J=4 \rightarrow 3)$ lines. The strength and slope of the emission are consistent with synchrotron emission from the radio-loud AGN. By averaging the line-free channels over the observed frequency range (LSB+USB for both lines) and fitting a two-dimensional Gaussian to the $u-v$ data, we find a $3.1 \mathrm{~mm}$ continuum flux of $3.61 \pm 0.18 \mathrm{mJy}$. In Figure 4, a map of the averaged continuum emission is shown. From the fit, we also find a continuum size of $\left(3^{\prime \prime} .14 \pm 2^{\prime \prime} .48\right) \times\left(0^{\prime \prime} .88 \pm 0^{\prime \prime} .72\right)$ at a position angle of $289^{\circ} \pm 19^{\circ}$, suggesting that the continuum emission is marginally resolved at best. Accounting for interferometric seeing (which is not part of the above errors), this is consistent with the $1^{\prime \prime} .7$ maximum image separation and the continuum emission to be dominated by the radio-loud AGN.

\subsubsection{Origin of the $\mathrm{CO} /$ Continuum Emission}

In Figure 10, overlays of the $\mathrm{CO}(J=4 \rightarrow 3)$ and underlying continuum emission on top of observed-frame $555 \mathrm{~nm}, 814 \mathrm{~nm}$, and $1.6 \mu \mathrm{m}$ continuum emission in B1359+154 are shown (HST images from Munoz et al. 1998). The peak of the $\mathrm{CO} /$ continuum emission is consistent with the centroid of the six lensed images of the source. Observations at (at least) $5 \times$ higher resolution are desirable to study the distribution and dynamics of the molecular gas in this complex system.

\section{DISCUSSION}

\subsection{The "Star Formation Law" for AGN Host Galaxies}

Due to high lensing magnification factors, our targets are among the intrinsically faintest high- $z$ sources observed to date.

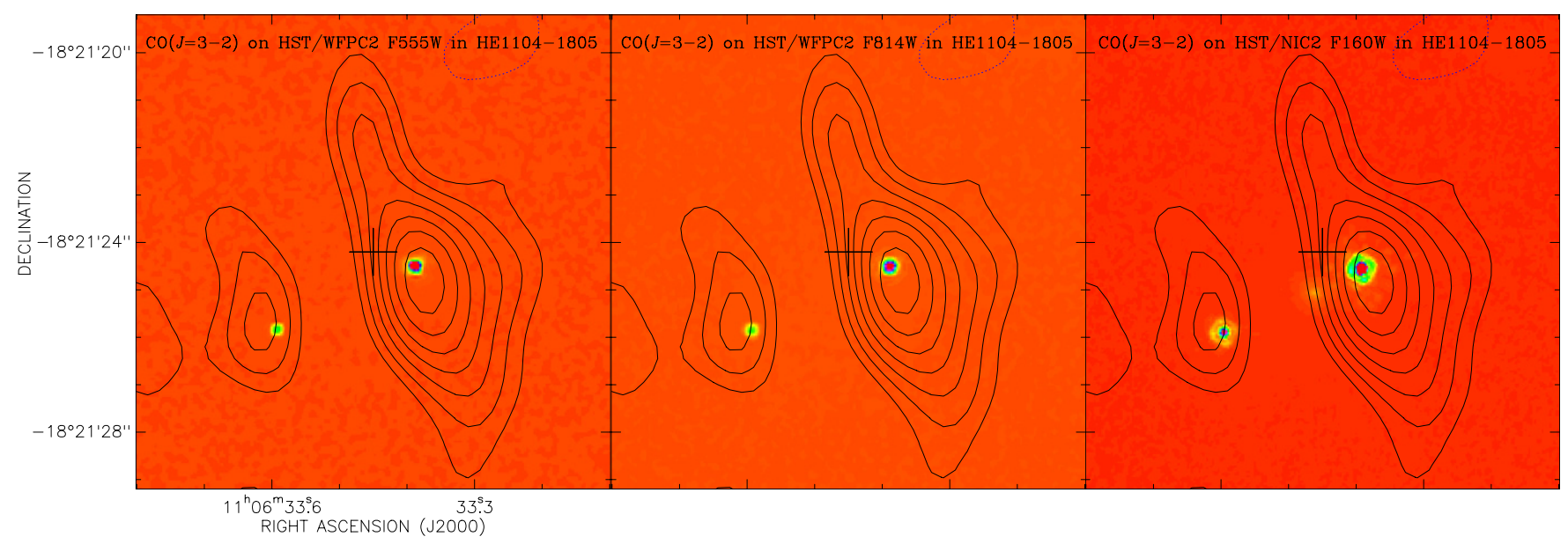

Figure 9. Overlays of $\mathrm{CO}(J=3 \rightarrow 2)$ (contours) and $555 \mathrm{~nm}, 814 \mathrm{~nm}$, and $1.6 \mu \mathrm{m}$ (rest-frame 167, 245, and $482 \mathrm{~nm}$ ) continuum emission (HST/WFPC2 and NICMOS2, left to right: images not cleaned) in HE 1104-1805. The cross indicates the same position as in Figure 7. The two brightest spots are the lens images of the background quasar, and the fainter spot in the middle is the foreground lensing galaxy.

(A color version of this figure is available in the online journal.) 


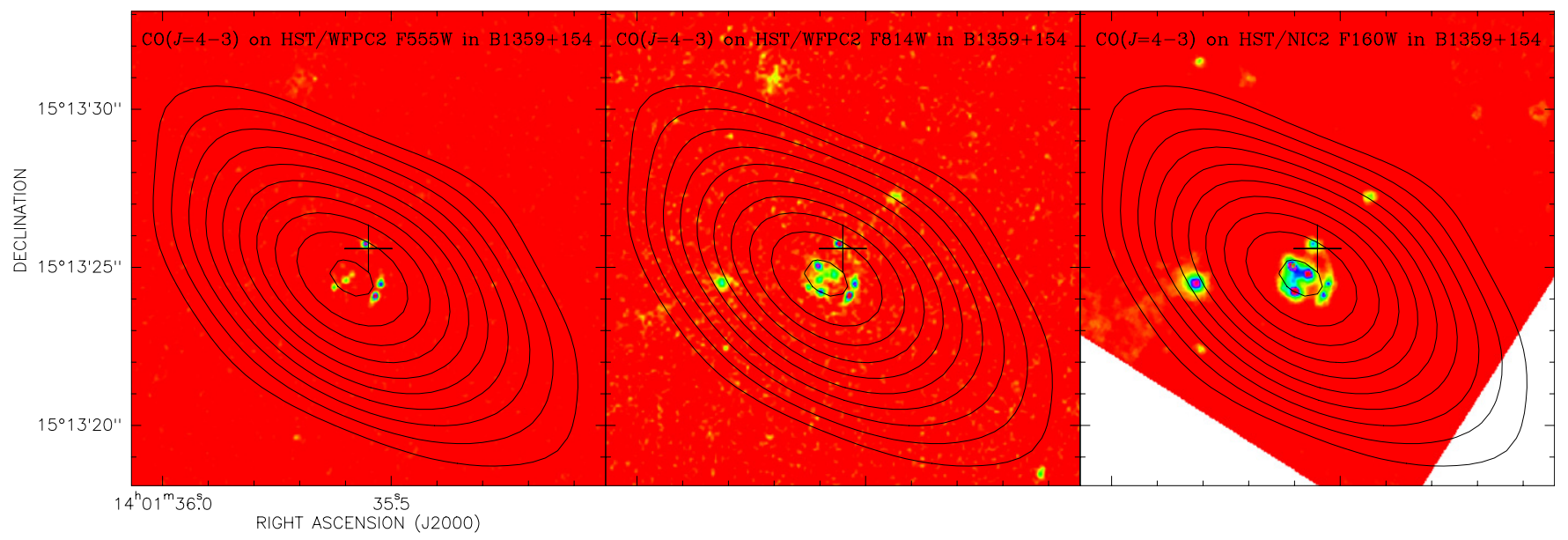

Figure 10. Overlays of $\mathrm{CO}(J=4 \rightarrow 3)$ (contours) and $555 \mathrm{~nm}, 814 \mathrm{~nm}$, and $1.6 \mu \mathrm{m}$ (rest-frame 131, 192, and $377 \mathrm{~nm}$ ) continuum emission $(H S T / \mathrm{WFPC} 2$ and NICMOS2, left to right: images not cleaned) in B1359+154. The cross indicates the same position as in Figure 7 . The six spots visible in all panels are the lens images of the background quasar, and the three additional spots in the central region that become visible at longer wavelengths are the foreground lensing galaxies.

(A color version of this figure is available in the online journal.)

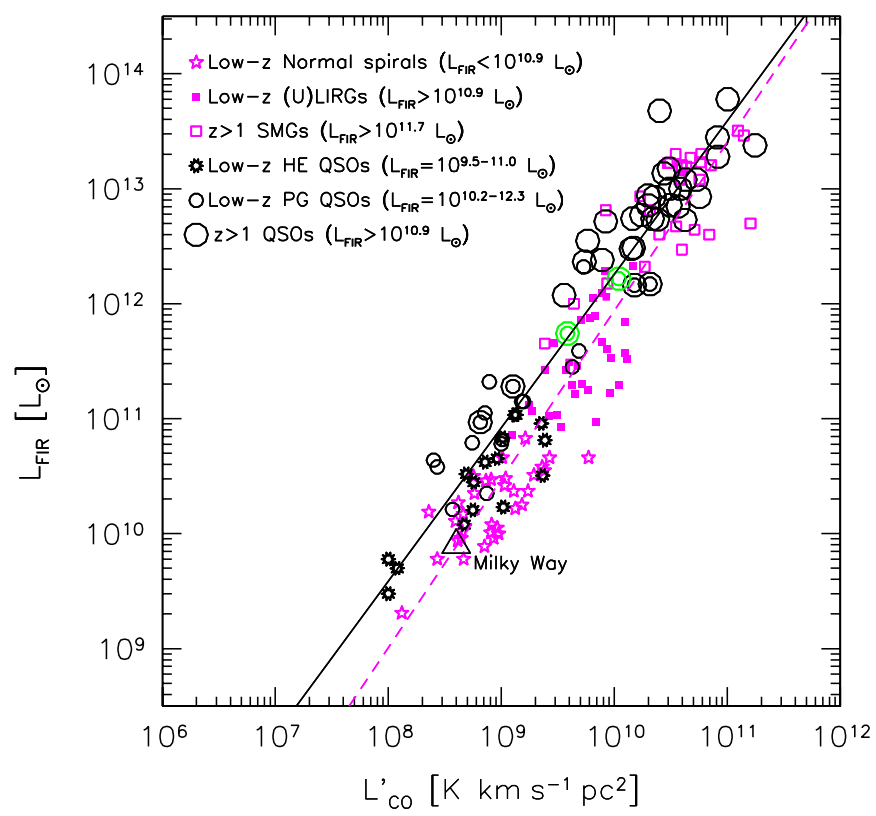

Figure 11. Comparison of $\mathrm{CO}$ line luminosity with FIR luminosity for samples of low- $z$ spiral and starburst galaxies and (U)LIRGs (Gao \& Solomon 2004), low- $z$ HE and PG QSOs (Evans et al. 2001, 2006; Scoville et al. 2003; Bertram et al. 2007), and high- $z$ SMGs (Solomon \& Vanden Bout 2005; Tacconi et al. 2006; Iono et al. 2006; Frayer et al. 2008, 2011; Schinnerer et al. 2008; Daddi et al. 2009a, 2009b; Knudsen et al. 2009; Weiß et al. 2009; Bothwell et al. 2010; Carilli et al. 2010; Riechers et al. 2010b; Harris et al. 2010) and QSOs (Solomon \& Vanden Bout 2005; Riechers et al. 2006, 2009a; Carilli et al. 2007; Maiolino et al. 2007; Willott et al. 2007; Aravena et al. 2008; Coppin et al. 2008; Wang et al. 2010). The new lensed high- $z$ QSOs in this paper are shown as double circles. For comparison, the green circles assume $\mu_{\mathrm{L}}=20$ for B1359+154 and B1938+666. All data are corrected for gravitational lensing. The solid line is a fit to all quasar samples (black symbols), corresponding to $\log L_{\mathrm{FIR}}=(1.33 \pm$ $0.05) \log L_{\mathrm{CO}}^{\prime}-(1.09 \pm 0.50)$. The dashed line is a fit to all sources without a dominant AGN (magenta symbols), corresponding to $\log L_{\mathrm{FIR}}=(1.47 \pm$ $0.06) \log L_{\mathrm{CO}}^{\prime}-(2.73 \pm 0.54)$. A fit to all data yields $L_{\mathrm{FIR}}=(1.40 \pm 0.04)$ $\log L_{\mathrm{CO}}^{\prime}-(1.92 \pm 0.42)$.

(A color version of this figure is available in the online journal.)

Their $L_{\mathrm{FIR}} / L_{\mathrm{CO}}^{\prime}$ ratios lie at the low end of the distribution of high- $z$ quasars, but are consistent with the observed range.

To investigate the properties of our targets in more detail, we here compare their $L_{\mathrm{CO}}^{\prime}$ and $L_{\mathrm{FIR}}$ to other CO-detected quasars at low and high redshifts, and low- and high-redshift galaxies without luminous AGNs; see Figure 11). The $L_{\mathrm{CO}}^{\prime}-L_{\mathrm{FIR}}$ relation is a variant of the spatially integrated "star formation law" (e.g., Kennicutt 1998a, 1998b) that only relies on observed quantities, as it is independent of quantities such as the conversion factor $\alpha_{\mathrm{CO}}$ and the assumed stellar initial mass function (see, e.g., Daddi et al. 2010a; Genzel et al. 2010, for recent investigations of the $M_{\mathrm{gas}}-\mathrm{SFR}$ relation). At high redshift, we restrict the analysis to a comparison of quasars (summarized in Table 4) and SMGs, as these samples cover a comparable range in $L_{\mathrm{FIR}}{ }^{7}$ Many SMGs are known to host AGNs, but these AGNs are substantially less luminous than those found in quasars. At low $z$, the galaxies without luminous AGNs are represented by a revised version of the Gao \& Solomon (2004) sample, including luminous and ULIRGs). This currently is the beststudied, best-calibrated low- $z$ galaxy sample for the spatially integrated galaxy properties studied here. We corrected all high$J$ CO line-based luminosities for excitation, based on models for high- $z$ quasars with well-sampled $\mathrm{CO}$ line ladders (Riechers et al. 2006, 2009b), corresponding to corrections of $0 \%, 2 \%$, $6 \%, 12 \%$, and $25 \%$ for the $\mathrm{CO} J=2 \rightarrow 1,3 \rightarrow 2,4 \rightarrow 3,5 \rightarrow 4$, and $6 \rightarrow 5$ lines. A fit to all data yields $L_{\mathrm{FIR}}=(1.40 \pm 0.04)$ $\log L_{\mathrm{CO}}^{\prime}-(1.92 \pm 0.42)$, yielding the canonical power-law slope of 1.4 (e.g., Kennicutt 1998a; see also Riechers et al. 2006). Fitting all quasar samples yields $\log L_{\mathrm{FIR}}=(1.33 \pm 0.05)$ $\log L_{\mathrm{CO}}^{\prime}-(1.09 \pm 0.50)$, i.e., a slightly lower power-law slope. A fit to the low $-z$ galaxy sample and the SMGs yields $\log L_{\mathrm{FIR}}=$ $(1.47 \pm 0.06) \log L_{\mathrm{CO}}^{\prime}-(2.73 \pm 0.54)$, i.e., a slightly higher power-law slope.

The high- $z$ quasars and SMG samples show comparable $L_{\mathrm{FIR}} /$ $L_{\mathrm{CO}}^{\prime}$ ratios and span a comparable range (see also Figure 12, where the ratio of both quantities is shown on a linear scale to emphasize the scatter of individual sources). On average, SMGs have somewhat higher ratios (by $\sim 28 \%$ at $L_{\mathrm{FIR}}=10^{13} L_{\odot}$ ), which is likely due to the fact that the typically $\mathrm{CO}(J=3 \rightarrow 2)$ based $L_{\mathrm{CO}}^{\prime}$ are undercorrected with the $2 \%$ correction factor for quasars. Recent studies of $\mathrm{CO}(J=1 \rightarrow 0)$ and $\mathrm{CO}(J=2 \rightarrow 1)$ emission in SMGs suggest that a correction factor of $30 \%-40 \%$

\footnotetext{
7 The high- $z$ massive, gas-rich star-forming galaxies identified by Daddi et al. (2008, 2010b) and Tacconi et al. (2010) have comparable $L_{\mathrm{CO}}^{\prime}$, but substantially lower $L_{\mathrm{FIR}} / L_{\mathrm{CO}}^{\prime}$, than these more extreme systems.
} 

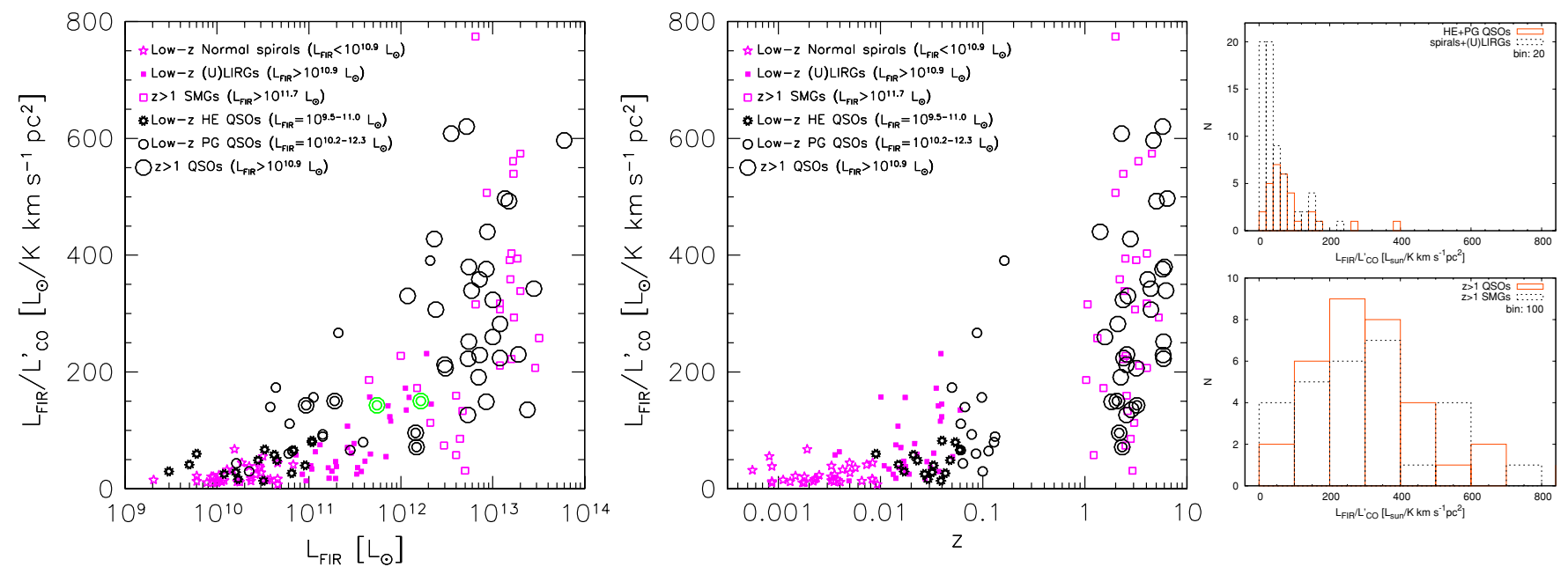

Figure 12. $L_{\mathrm{FIR}} / L_{\mathrm{CO}}^{\prime}$, a measure of star formation efficiency, as a function of $L_{\mathrm{FIR}}$ (left) and redshift (middle), and as histograms for the low- $z$ and high- $z$ samples (right). The ratio is shown on a linear scale to emphasize the scatter of the $L_{\mathrm{CO}}^{\prime}-L_{\mathrm{FIR}}$ relation. The same samples are shown as in Figure 11.

(A color version of this figure is available in the online journal.)

Table 4

Molecular Line and FIR Continuum Properties of High- $z$ Quasars

\begin{tabular}{|c|c|c|c|c|c|c|c|c|}
\hline Source & $z_{\mathrm{CO}}$ & $\mu_{\mathrm{L}}$ & CO Line & $\begin{array}{c}I_{\mathrm{CO}} \\
\left(\mathrm{Jy} \mathrm{km} \mathrm{s}^{-1}\right)\end{array}$ & $\begin{array}{l}\log _{10}\left(L_{\mathrm{CO}}^{\prime}\right)^{\mathrm{a}, \mathrm{b}} \\
\left(\mathrm{K} \mathrm{km} \mathrm{s}^{-1} \mathrm{pc}^{2}\right)\end{array}$ & $\begin{array}{c}\log _{10}\left(L_{\mathrm{FIR}}\right)^{\mathrm{a}} \\
\left(L_{\odot}\right)\end{array}$ & Ref. & Comments \\
\hline Q0957+561 & 1.4141 & 1.6 & $2 \rightarrow 1$ & $1.2 \pm 0.1$ & 10.30 & 12.94 & 1 & Double lens \\
\hline $3 \mathrm{C} 318$ & 1.571 & & $2 \rightarrow 1$ & $1.19 \pm 0.22$ & 10.59 & 13.00 & 2 & \\
\hline COSBO11 & 1.8275 & & $2 \rightarrow 1$ & 1.33 & 10.76 & 12.93 & 3 & \\
\hline B1938+666 & 2.0590 & 173 & $3 \rightarrow 2$ & $9.1 \pm 1.1$ & 9.10 & 11.28 & 4 & Einstein ring \\
\hline HS $1002+4400$ & 2.1015 & & $3 \rightarrow 2$ & $1.7 \pm 0.3$ & 10.63 & 13.08 & 5 & \\
\hline HE $0230-2130$ & 2.1664 & 14.5 & $3 \rightarrow 2$ & $8.3 \pm 1.2$ & 10.18 & 12.16 & 4 & Quadruple lens \\
\hline RX J1249-0559 & 2.2470 & & $3 \rightarrow 2$ & $1.3 \pm 0.4$ & 10.56 & 12.85 & 5 & \\
\hline F10214+4724 & 2.2858 & 17 & $3 \rightarrow 2$ & $3.40 \pm 0.19$ & 9.76 & 12.55 & 6 & Arc lens \\
\hline HE $1104-1805$ & 2.3221 & 10.8 & $3 \rightarrow 2$ & $7.5 \pm 1.2$ & 10.32 & 12.17 & 4 & Double lens \\
\hline $\mathrm{J} 1543+5359$ & 2.3698 & & $3 \rightarrow 2$ & $1.0 \pm 0.2$ & 10.49 & 13.00 & 5 & \\
\hline HS $1611+4719$ & 2.3961 & & $3 \rightarrow 2$ & $1.7 \pm 0.3$ & 10.73 & 13.08 & 5 & \\
\hline $\mathrm{J} 13122+12381$ & 2.5564 & & $3 \rightarrow 2$ & $0.4 \pm 0.1$ & 10.15 & 12.48 & 5 & \\
\hline Cloverleaf & 2.55784 & 11 & $3 \rightarrow 2$ & $13.2 \pm 0.2$ & 10.63 & 12.73 & 7 & Quadruple lens \\
\hline $\mathrm{J} 1409+5628$ & 2.5832 & & $3 \rightarrow 2$ & $2.3 \pm 0.2$ & 10.92 & 13.28 & 8 & \\
\hline MG 0414+0534 & 2.639 & 27 & $3 \rightarrow 2$ & 2.6 & 9.55 & 12.07 & 9 & Quadruple lens \\
\hline RX J0911+0551 & 2.796 & 22 & $3 \rightarrow 2$ & $2.9 \pm 1.1$ & 9.73 & 12.37 & 10 & Quadruple lens \\
\hline J04135+10277 & 2.846 & 1.3 & $3 \rightarrow 2$ & $5.4 \pm 1.3$ & 11.25 & 13.38 & 10 & Cluster lens \\
\hline MG $0751+2716$ & 3.1999 & 16 & $3 \rightarrow 2$ & $4.6 \pm 0.5$ & 10.17 & 12.49 & 11 & Quadruple lens \\
\hline B1359+154 & 3.2399 & 118 & $4 \rightarrow 3$ & $2.5 \pm 0.4$ & 8.81 & 10.97 & 4 & Sextuple lens \\
\hline APM $08279+5255$ & 3.9118 & 4.2 & $1 \rightarrow 0$ & $0.168 \pm 0.015$ & 10.40 & 13.68 & 12 & Triple lens \\
\hline PSS J2322+1944 & 4.1192 & 5.3 & $1 \rightarrow 0$ & $0.155 \pm 0.013$ & 10.30 & 12.86 & 13 & Einstein ring \\
\hline BRI 1335-0417 & 4.4074 & & $2 \rightarrow 1$ & $0.43 \pm 0.02$ & 10.91 & 13.45 & 14 & \\
\hline BRI 0952-0115 & 4.4337 & 4 & $5 \rightarrow 4$ & $0.91 \pm 0.11$ & 9.89 & 12.38 & 15 & Double lens \\
\hline BR 1202-0725 & 4.6949 & & $1 \rightarrow 0$ & $0.120 \pm 0.010$ & 11.00 & 13.78 & 13 & Two sources \\
\hline J0338+0021 & 5.0267 & & $5 \rightarrow 4$ & $0.73 \pm 0.09$ & 10.48 & 13.18 & 16 & \\
\hline J0927+2001 & 5.7722 & & $5 \rightarrow 4$ & $0.44 \pm 0.07$ & 10.35 & 12.93 & 17 & \\
\hline $\mathrm{J} 1044-0125$ & 5.7824 & & $6 \rightarrow 5$ & $0.21 \pm 0.04$ & 9.92 & 12.72 & 18 & \\
\hline J0840+5624 & 5.8437 & & $5 \rightarrow 4$ & $0.60 \pm 0.07$ & 10.50 & 12.86 & 18 & \\
\hline $\mathrm{J} 1425+3254$ & 5.8918 & & $6 \rightarrow 5$ & $0.59 \pm 0.11$ & 10.38 & 12.73 & 18 & \\
\hline $\mathrm{J} 1335+3533$ & 5.9012 & & $6 \rightarrow 5$ & $0.53 \pm 0.07$ & 10.34 & 12.74 & 18 & \\
\hline J2054-0005 & 6.0379 & & $6 \rightarrow 5$ & $0.34 \pm 0.07$ & 10.16 & 12.74 & 18 & \\
\hline $\mathrm{J} 1048+4637$ & 6.2274 & & $6 \rightarrow 5$ & $0.39 \pm 0.08$ & 10.24 & 12.77 & 18 & \\
\hline $\mathrm{J} 1148+5251$ & 6.4189 & & $3 \rightarrow 2$ & $0.20 \pm 0.02$ & 10.48 & 13.13 & 19 & \\
\hline
\end{tabular}

Notes. Estimates are typically based on the lowest- $J$ CO line observed, if applicable.

${ }^{a}$ Corrected for gravitational lensing.

${ }^{\mathrm{b}}$ Corrected for line excitation where applicable. Corrections applied: $0 \%$ for $\mathrm{CO}(J=2 \rightarrow 1), 2 \%$ for $\mathrm{CO}(J=3 \rightarrow 2), 6 \%$ for $\mathrm{CO}(J=4 \rightarrow 3), 12 \%$ for $\mathrm{CO}(J=$ $5 \rightarrow 4)$, and $25 \%$ for $\mathrm{CO}(J=6 \rightarrow 5)$.

References. (1) Krips et al. 2005; (2) Willott et al. 2007; (3) Aravena et al. 2008; (4) this work; (5) Coppin et al. 2008; (6) Ao et al. 2008; (7) Weiß et al. 2003; (8) Beelen et al. 2004; (9) Barvainis et al. 1998; (10) Hainline et al. 2004; (11) Alloin et al. 2007; (12) Riechers et al. 2009a; (13) Riechers et al. 2006; (14) Riechers et al. 2008; (15) Guilloteau et al. 1999; (16) Maiolino et al. 2007; (17) Carilli et al. 2007; (18) Wang et al. 2010; (19) Walter et al. 2003. 
may be more appropriate for a substantial fraction of SMGs (e.g., Ivison et al. 2010; Carilli et al. 2010; Riechers et al. 2010b; Harris et al. 2010; Frayer et al. 2011), which would make the $L_{\mathrm{FIR}} / L_{\mathrm{CO}}^{\prime}$ ratios for quasars and SMGs even more similar on average. However, studies of the $\mathrm{CO}$ excitation in SMGs also show that the emission seen in low- $J$ lines may partially be associated with a low-excitation gas component, i.e., material that likely is not concentrated in the regions where the massive starbursts take place (Carilli et al. 2010; Riechers et al. 2010b). This is not the case in quasars, which can typically be modeled with a single, highly excited gas component down to the low-J CO lines (e.g., Riechers et al. 2006; Weiß et al. 2007). Studies of $\mathrm{CO}(J=1 \rightarrow 0)$ emission in larger samples of high- $z$ quasars and SMGs are required to investigate these differences, which directly affect the physical interpretation of the $L_{\mathrm{CO}}^{\prime}-L_{\mathrm{FIR}}$ relation. In fact, observations of $\mathrm{CO}$ line ladders down to $\mathrm{CO}(J=$ $1 \rightarrow 0$ ) may be a powerful tool to distinguish different high- $z$ galaxy populations based on their gas properties alone (e.g., Dannerbauer et al. 2009; Aravena et al. 2010; Riechers et al. 2010a).

Another important aspect is that here we study $L_{\text {FIR }}$ (i.e., rest-frame 42.5-122.5 $\mu \mathrm{m}$ emission), not $L_{\mathrm{IR}}(8-1000 \mu \mathrm{m})$. This has a relatively small impact on galaxies without luminous AGNs, but it makes a substantial difference for quasars. Quasars typically show both a warm dust bump with temperatures of $T_{\text {dust }}=30-60 \mathrm{~K}$ (i.e., comparable to ULIRGs and SMGs) and a hot dust bump with $T_{\text {dust }}>100 \mathrm{~K}$ (likely associated with the AGN, e.g., Weiß et al. 2003; Beelen et al. 2006). This yields an $L_{\mathrm{IR}} / L_{\mathrm{FIR}}$ ratio of typically a factor of a few. Thus, the $L_{\mathrm{CO}}^{\prime}-L_{\mathrm{IR}}$ relation for quasars would likely be biased toward the AGN properties. On the other hand, our findings for the $L_{\mathrm{CO}}^{\prime}-L_{\mathrm{FIR}}$ relation appear to suggest that, indeed, the FIR luminosity in high- $z$ quasars is dominated by dust-reprocessed emission from young stars in the host galaxy, rather than the AGN.

One caveat to the present interpretation of the $L_{\mathrm{CO}}^{\prime}-L_{\mathrm{FIR}}$ relation at high- $z$ is the possibility of differential gravitational lensing effects between the $\mathrm{CO}$ and FIR emission for the lensed galaxies in both samples. Given the overall agreement between lensed and unlensed sources within the samples and the fact that they follow the $L_{\mathrm{CO}}^{\prime}-L_{\mathrm{FIR}}$ relation, such effects appear to be minor, but only a few lensed systems have been studied at sufficient spatial resolution in the molecular gas and FIR continum emission to quantify this in more detail.

The difference in $L_{\mathrm{FIR}} / L_{\mathrm{CO}}^{\prime}$ ratios between low- $z$ quasar samples and low $-z$ galaxies without luminous AGNs appear to be larger than those between high- $z$ quasars and SMGs. With the current sample sizes, these differences are only moderately statistically significant. Given the less massive dust and gas reservoirs in the lower luminosity systems, such a trend may however not be surprising. In such systems, the AGN may be substantially more efficient in heating larger fractions of the warm dust than in the typically very gas-rich high- $z$ systems, yielding elevated $L_{\mathrm{FIR}} / L_{\mathrm{CO}}^{\prime}$ ratios. High spatial resolution observations of the $\mathrm{CO}$ and FIR continuum in nearby quasar host galaxies are key for investigating this issue in more detail. An alternative interpretation would be that the AGN fraction rises with $L_{\text {FIR }}$ toward ULIRGs and SMGs, which could decrease the difference between FIR-luminous quasars and other FIR-luminous galaxy populations. We consider this explanation less compelling, as there is no clear relation between AGN luminosity and FIR luminosity for galaxies of comparable masses.

\subsection{Star Formation Rates and Gas Depletion Timescales}

If the $L_{\text {FIR }}$ in our targets are indeed dominated by star formation, we can derive their SFRs. Assuming SFR $\left[M_{\odot} \mathrm{yr}^{-1}\right]=$ $1.5 \times 10^{-10} L_{\mathrm{FIR}}\left[L_{\odot}\right]$ (e.g., Kennicutt 1998a, 1998b), we find SFRs of $30\left(\mu_{\mathrm{L}} / 173\right)^{-1} M_{\odot} \mathrm{yr}^{-1}, 220\left(\mu_{\mathrm{L}} / 14.5\right)^{-1} M_{\odot} \mathrm{yr}^{-1}$, $220\left(\mu_{\mathrm{L}} / 10.8\right)^{-1} M_{\odot} \mathrm{yr}^{-1}$, and $14\left(\mu_{\mathrm{L}} / 118\right)^{-1} M_{\odot} \mathrm{yr}^{-1}$ for B1938+666, HE 0230-2130, HE 1104-1805, and B1359+154, respectively (Table 3 ).

The minimum times for which the starbursts can be maintained at their current rates are given by the gas depletion timescales. We find $\tau_{\text {dep }}=M_{\text {gas }} / \mathrm{SFR}=35,55,75$, and 40 Myr for B1938+666, HE 0230-2130, HE 1104-1805, and $\mathrm{B} 1359+154$, respectively. This is comparable to what is found in SMGs and other high-z QSOs (e.g., Greve et al. 2005; Riechers et al. 2008, 2010b).

\subsection{Systemic Redshifts}

We find that the $\mathrm{CO}$ redshifts of the three sources with spectroscopic redshifts from optical observations are close to $z_{\text {opt }}$. However, in all cases, the $\mathrm{CO}$ emission is redshifted relative to broad $\operatorname{Ly} \alpha$ and high ionization (typically C IV $\lambda 1549$ ) optical emission lines from the AGN. The median redshift difference of the three sources with $z_{\text {opt }}$ is $d z=0.0045 \pm 0.0015$, or $360 \pm 116 \mathrm{~km} \mathrm{~s}^{-1} .8$ For a large sample of Sloan Digital Sky Survey quasars, Richards et al. (2002) have found that lowionization lines such as $\mathrm{Mg} \mathrm{II}(\lambda 2798)$ are commonly redshifted relative to high-ionization broad lines such as $\operatorname{C} \operatorname{IV}(\lambda 1549)$ (median of $824 \pm 511 \mathrm{~km} \mathrm{~s}^{-1}$ ), which they interpret to be due to orientation effects related to the AGN accretion disk (or disk wind). Even though the orientation of the accretion disk and host galaxy needs not be aligned, referencing the redshifts of broad AGN lines to the systemic redshifts provided by $\mathrm{CO}$ observations of the host galaxies may help to better disentangle orientation effects and dynamics of high-redshift galaxies.

\section{SUMMARY}

We have detected $\mathrm{CO}(J=2 \rightarrow 1), \mathrm{CO}(J=3 \rightarrow 2)$, and $\mathrm{CO}(J=$ $4 \rightarrow 3$ ) emission in four lensed quasar host galaxies at redshifts of $z>2$ with CARMA (a total of six lines). To date, these are the highest redshift $\mathrm{CO}$ detections reported with CARMA. From our analysis, we obtain the following key results.

Facilitating the large, $>7.4 \mathrm{GHz}$ bandwidth (LSB+USB) of the new CARMA correlator, we have executed the first successful "blind" CO redshift search with an interferometer, demonstrating the feasibility of such investigations with only few frequency setups. Based on the CO redshift, we also used the broad frequency range covered by these observations to search for emission from dense molecular gas tracers ( $\mathrm{HCN}$, $\mathrm{HCO}^{+}, \mathrm{HNC}, \mathrm{C}_{2} \mathrm{H}$, and $\mathrm{CN}$ ), which yielded only a statistical detection after stacking - as expected at the depth of this search. We also searched for $\mathrm{HCN}, \mathrm{HCO}^{+}$, and $\mathrm{HNC}$ absorption toward foreground sources, but no lines are detected along the line of sight.

We spatially resolve the $\mathrm{CO}$ emission in the two radioquiet quasars in the sample. In the wide separation double lens HE 1104-1805 $(z=2.322)$, we individually detect the emission toward both images. In all cases, the $\mathrm{CO}$ emission is consistent

\footnotetext{
8 For consistency, we here adopt $z_{\mathrm{Ly} \alpha}=2.3172$ for HE $1104-1805$ as measured by Smette et al. (1995) instead of the $\mathrm{H} \beta+[\mathrm{O} \mathrm{III}](\lambda 5007)$ redshift used above.
} 
with the brightest emission regions detected at rest-frame optical wavelengths.

We detect strong continuum emission toward the two radioloud sources in the sample. The spectral slope of the emission is consistent with synchrotron emission associated with the AGN in both cases.

We derive lensing- and excitation-corrected $\mathrm{CO}$ line luminosities of $0.65-21 \times 10^{9} \mathrm{~K} \mathrm{~km} \mathrm{~s}^{-1} \mathrm{pc}^{2}$. From the CO luminosities, we determine molecular gas masses of $0.52-17 \times 10^{9} \mathrm{M}_{\odot}$. These values are at the lower end of those observed in other high- $z$ quasars, showing the advantage of observing lensed distant galaxies to probe down to lower gas masses.

Combining our targets with literature samples, we find no significant difference in the $L_{\mathrm{FIR}} / L_{\mathrm{CO}}^{\prime}$ ratios between high$z$ quasars and SMGs. We find tentative evidence that nearby quasars with low $L_{\mathrm{FIR}}$ show an excess in $L_{\mathrm{FIR}} / L_{\mathrm{CO}}^{\prime}$ relative to systems without a luminous AGN at similar redshift and with comparable $L_{\mathrm{CO}}^{\prime}$. This may indicate that AGN heating of the warm dust is more efficient than in the more gas-rich and massive FIR-luminous quasars detected at high $z$, where the dust heating in the FIR appears to be largely dominated by young stars.

The observations presented herein lay the foundation for future studies with the Atacama Large (sub)Millimeter Array (ALMA), which will routinely observe unlensed galaxies at comparable and fainter intrinsic CO luminosities. The "blind" $\mathrm{CO}$ redshift search technique presented here will be particularly valuable for so-called Deep Field investigations with ALMA, which will allow us, for the first time, to construct an unbiased CO luminosity function out to high- $z$ by directly selecting galaxies through their $\mathrm{CO}$ content. This is an important step toward constraining the gas mass history of the universe, a critical piece in our understanding of galaxy evolution throughout cosmic times.

We thank the referee for a helpful and constructive report. D.R. acknowledges support from NASA through Hubble Fellowship grant HST-HF-51235.01 awarded by the Space Telescope Science Institute, which is operated by the Association of Universities for Research in Astronomy, Inc., for NASA, under contract NAS 5-26555. Support for CARMA construction was derived from the G. and B. Moore Foundation, the K. T. and E. L. Norris Foundation, the Associates of the California Institute of Technology, the states of California, Illinois, and Maryland, and the NSF. Ongoing CARMA development and operations are supported by the NSF under a cooperative agreement, and by the CARMA partner universities.

\section{REFERENCES}

Alloin, D., Kneib, J.-P., Guilloteau, S., \& Bremer, M. 2007, A\&A, 470, 53

Anguita, T., Faure, C., Yonehara, A., Wambsganss, J., Kneib, J.-P., Covone, G., \& Alloin, D. 2008, A\&A, 481, 615

Ao, Y., Weiß, A., Downes, D., Walter, F., Henkel, C., \& Menten, K. M. 2008, A\&A, 491, 747

Aravena, M., et al. 2008, A\&A, 491, 173

Aravena, M., et al. 2010, ApJ, 718, 177

Barvainis, R., Alloin, D., Guilloteau, S., \& Antonucci, R. 1998, ApJ, 492, L13

Barvainis, R., \& Ivison, R. J. 2002, ApJ, 571, 712

Beelen, A., Cox, P., Benford, D. J., Dowell, C. D., Kovács, A., Bertoldi, F., Omont, A., \& Carilli, C. L. 2006, ApJ, 642, 694

Beelen, A., et al. 2004, A\&A, 423, 441

Bertram, T., Eckart, A., Fischer, S., Zuther, J., Straubmeier, C., Wisotzki, L., \& Krips, M. 2007, A\&A, 470, 571

Bothwell, M. S., et al. 2010, MNRAS, 405, 219

Carilli, C. L., et al. 2007, ApJ, 666, L9

Carilli, C. L., et al. 2010, ApJ, 714, 1407
Coppin, K. E. K., et al. 2008, MNRAS, 389, 45

Daddi, E., Dannerbauer, H., Elbaz, D., Dickinson, M., Morrison, G., Stern, D., \& Ravindranath, S. 2008, ApJ, 673, L21

Daddi, E., et al. 2009a, ApJ, 694, 1517

Daddi, E., et al. 2009b, ApJ, 695, L176

Daddi, E., et al. 2010a, ApJ, 714, L118

Daddi, E., et al. 2010b, ApJ, 713, 686

Dannerbauer, H., Daddi, E., Riechers, D. A., Walter, F., Carilli, C. L., Dickinson, M., Elbaz, D., \& Morrison, G. E. 2009, ApJ, 698, L178

Downes, D., \& Solomon, P. M. 1998, ApJ, 507, 615

Eigenbrod, A., Courbin, F., Meylan, G., Vuissoz, C., \& Magain, P. 2006, A\&A, 451, 759

Evans, A. S., Frayer, D. T., Surace, J. A., \& Sanders, D. B. 2001, AJ, 121, 1893

Evans, A. S., Solomon, P. M., Tacconi, L. J., Vavilkin, T., \& Downes, D. 2006, AJ, 132, 2398

Frayer, D. T., et al. 2008, ApJ, 680, L21

Frayer, D. T., et al. 2011, ApJ, 726, L22

Gao, Y., Carilli, C. L., Solomon, P. M., \& Vanden Bout, P. A. 2007, ApJ, 660, L93

Gao, Y., \& Solomon, P. M. 2004, ApJ, 606, 271

Genzel, R., et al. 2010, MNRAS, 407, 2091

Greve, T. R., et al. 2005, MNRAS, 359, 1165

Guilloteau, S., Omont, A., Cox, P., McMahon, R. G., \& Petitjean, P. 1999, A\&A, 349,363

Hainline, L. J., Scoville, N. Z., Yun, M. S., Hawkins, D. W., Frayer, D. T., \& Isaak, K. G. 2004, ApJ, 609, 61

Harris, A. I., Baker, A. J., Zonak, S. G., Sharon, C. E., Genzel, R., Rauch, K., Watts, G., \& Creager, R. 2010, ApJ, 723, 1139

Iono, D., et al. 2006, PASJ, 58, 957

Ivison, R. J., Smail, I., Papadopoulos, P. P., Wold, I., Richard, J., Swinbank, A. M., Kneib, J.-P., \& Owen, F. N. 2010, MNRAS, 404, 198

Kennicutt, R. C. 1998a, ApJ, 498, 541

Kennicutt, R. C. 1998b, ARA\&A, 36, 189

King, L. J., Browne, I. W. A., Muxlow, T. W. B., Narasimha, D., Patnaik, A. R., Porcas, R. W., \& Wilkinson, P. N. 1997, MNRAS, 289, 450

King, L. J., et al. 1998, MNRAS, 295, L41

Knudsen, K. K., Neri, R., Kneib, J.-P., \& van der Werf, P. P. 2009, A\&A, 496, 45

Krips, M., Neri, R., Eckart, A., Downes, D., Martín-Pintado, J., \& Planesas, P. 2005, A\&A, 431, 879

Lehár, J., et al. 2000, ApJ, 536, 584

Lidman, C., Courbin, F., Kneib, J.-P., Golse, G., Castander, F., \& Soucail, G. 2000, A\&A, 364, L62

Lutz, D., et al. 2008, ApJ, 684, 853

Magain, P., Surdej, J., Swings, J.-P., Borgeest, U., \& Kayser, R. 1988, Nature, 334, 325

Maiolino, R., et al. 2007, A\&A, 472, L33

Munoz, J. A., Falco, E. E., Kochanek, C. S., Lehár, J., McLeod, B. A., Impey, C. D., Rix, H.-W., \& Peng, C. Y. 1998, Ap\&SS, 263, 51

Myers, S. T., et al. 1999, AJ, 117, 2565

Omont, A., Beelen, A., Bertoldi, F., Cox, P., Carilli, C. L., Priddey, R. S., McMahon, R. G., \& Isaak, K. G. 2003, A\&A, 398, 857

Omont, A., Cox, P., Bertoldi, F., McMahon, R. G., Carilli, C., \& Isaak, K. G. 2001, A\&A, 374, 371

Patnaik, A. R., Browne, I. W. A., Wilkinson, P. N., \& Wrobel, J. M. 1992 MNRAS, 254, 655

Peng, C. Y., Impey, C. D., Rix, H.-W., Kochanek, C. S., Keeton, C. R., Falco, E. E., Lehár, J., \& McLeod, B. A. 2006, ApJ, 649, 616

Remy, M., Claeskens, J.-F., Surdej, J., Hjorth, J., Refsdal, S., Wucknitz, O., Sorensen, A. N., \& Grundahl, F. 1998, New Astron., 3, 379

Richards, G. T., Vanden Berk, D. E., Reichard, T. A., Hall, P. B., Schneider, D. P., SubbaRao, M., Thakar, A. R., \& York, D. G. 2002, AJ, 124, 1

Riechers, D. A., Carilli, C. L., Walter, F., \& Momjian, E. 2010a, ApJ, 724, L153

Riechers, D. A., Walter, F., Carilli, C. L., \& Bertoldi, F. 2007a, ApJ, 671, L13

Riechers, D. A., Walter, F., Carilli, C. L., Bertoldi, F., \& Momjian, E. 2008, ApJ, 686, L9

Riechers, D. A., Walter, F., Carilli, C. L., \& Lewis, G. F. 2009a, ApJ, 690, 463

Riechers, D. A., Walter, F., Cox, P., Carilli, C. L., Weiss, A., Bertoldi, F., \& Neri, R. 2007b, ApJ, 666, 778

Riechers, D. A., et al. 2006, ApJ, 650, 604

Riechers, D. A., et al. 2009b, ApJ, 703, 1338

Riechers, D. A., et al. 2010b, ApJ, 720, L131

Rusin, D., et al. 2001, ApJ, 557, 594

Sanders, D. B., Scoville, N. Z., \& Soifer, B. T. 1991, ApJ, 370, 158

Schinnerer, E., et al. 2008, ApJ, 689, L5

Scoville, N. Z., Frayer, D. T., Schinnerer, E., \& Christopher, M. 2003, ApJ, 585, L105 
Smette, A., Robertson, J. G., Shaver, P. A., Reimers, D., Wisotzki, L., \& Koehler, T. 1995, A\&AS, 113, 199

Solomon, P. M., \& Vanden Bout, P. A. 2005, ARA\&A, 43, 677

Spergel, D. N., et al. 2003, ApJS, 148, 175

Spergel, D. N., et al. 2007, ApJS, 170, 377

Sulentic, J. W., Repetto, P., Stirpe, G. M., Marziani, P., Dultzin-Hacyan, D., \& Calvani, M. 2006, A\&A, 456, 929

Tacconi, L. J., et al. 2006, ApJ, 640, 228

Tacconi, L. J., et al. 2010, Nature, 463, 781

Tonry, J. L., \& Kochanek, C. S. 2000, AJ, 119, 1078

Venturini, S., \& Solomon, P. M. 2003, ApJ, 590, 740

Walter, F., et al. 2003, Nature, 424, 406
Wang, R., et al. 2008, ApJ, 687, 848

Wang, R., et al. 2010, ApJ, 714, 699

Weiß, A., Henkel, C., Downes, D., \& Walter, F. 2003, A\&A, 409, L41

Weiß, A., Ivison, R. J., Downes, D., Walter, F., Cirasuolo, M., \& Menten, K. M. 2009, ApJ, 705, L45

Weiß, A., et al. 2007, A\&A, 467, 955

Wiklind, T., \& Combes, F. 1995, A\&A, 299, 382

Wiklind, T., \& Combes, F. 1996, Nature, 379, 139

Willott, C. J., Martínez-Sansigre, A., \& Rawlings, S. 2007, AJ, 133, 564

Wisotzki, L., Christlieb, N., Liu, M. C., Maza, J., Morgan, N. D., \& Schechter, P. L. 1999, A\&A, 348, L41

Wisotzki, L., Koehler, T., Ikonomou, M., \& Reimers, D. 1995, A\&A, 297, L59 